\title{
Spectroscopic and Structural Probing of Excited-State Molecular Dynamics with Time-Resolved Photoelectron Spectroscopy and Ultrafast Electron Diffraction
}

\author{
Yusong Liu $\odot,{ }^{1}$ Spencer L. Horton, ${ }^{1}$ Jie Yang, ${ }^{2,3}$ J. Pedro F. Nunes $\odot,{ }^{5}$ Xiaozhe Shen, ${ }^{2}$ Thomas J. A. Wolf $\odot,{ }^{2,3}$ \\ Ruaridh Forbes, ${ }^{2,3,6,7}$ Chuan Cheng, ${ }^{1}$ Bryan Moore, ${ }^{5}$ Martin Centurion, ${ }^{5}$ Kareem Hegazy, ${ }^{3,4}$ Renkai Li, ${ }^{2}$ \\ Ming-Fu Lin, ${ }^{2}$ Albert Stolow, ${ }^{7,8,9}$ Paul Hockett@ ${ }^{9}$ Tamás Rozgonyi, ${ }^{10,11}$ Philipp Marquetand $\odot,{ }^{12,13,14, *}$ \\ Xijie Wang, ${ }^{2, \dagger}$ and Thomas Weinacht ${ }^{1, \dagger}$ \\ ${ }^{1}$ Department of Physics and Astronomy, Stony Brook University, Stony Brook, New York 11794, USA \\ ${ }^{2}$ SLAC National Accelerator Laboratory, Menlo Park, California 94025, USA \\ ${ }^{3}$ Stanford PULSE Institute, SLAC National Accelerator Laboratory, Menlo Park, California 94025, USA \\ ${ }^{4}$ Department of Physics, Stanford University, Stanford, California 94305, USA \\ ${ }^{5}$ Department of Physics and Astronomy, University of Nebraska-Lincoln, Lincoln, Nebraska 68588, USA \\ ${ }^{6}$ Department of Physics and Astronomy, University College London, \\ Gower Street, London, WC1E 6BT, United Kingdom \\ ${ }^{7}$ Department of Physics, University of Ottawa, 150 Louis Pasteur, Ottawa, Ontario K1N 6N5, Canada \\ ${ }^{8}$ Department of Chemistry, University of Ottawa, 10 Marie Curie, Ottawa, Ontario K1N 6N5, Canada \\ ${ }^{9}$ National Research Council of Canada, 100 Sussex Drive, Ottawa, Ontario K1A OR6, Canada \\ ${ }^{10}$ Institute of Materials and Environmental Chemistry, Research Centre for Natural Sciences, Hungarian \\ Academy of Sciences, Budapest 1117 Magyar tudósok körútja 2, Hungary \\ ${ }^{11}$ Wigner Research Centre for Physics, Hungarian Academy of Sciences, \\ P.O. Box 49, H-1525 Budapest, Hungary \\ ${ }^{12}$ University of Vienna, Faculty of Chemistry, Institute of Theoretical Chemistry, \\ Währinger Straße 17, 1090 Wien, Austria \\ ${ }^{13}$ Vienna Research Platform on Accelerating Photoreaction Discovery, University of Vienna, Währinger \\ Straße 17, 1090 Wien, Austria \\ ${ }^{14}$ University of Vienna, Faculty of Chemistry, Data Science at University of Vienna, Währinger Straße 29, \\ 1090 Wien, Austria
}

(Received 13 July 2019; revised manuscript received 19 February 2020; accepted 5 March 2020; published 22 April 2020)

\begin{abstract}
Pump-probe measurements aim to capture the motion of electrons and nuclei on their natural timescales (femtoseconds to attoseconds) as chemical and physical transformations take place, effectively making "molecular movies" with short light pulses. However, the quantum dynamics of interest are filtered by the coordinate-dependent matrix elements of the chosen experimental observable. Thus, it is only through a combination of experimental measurements and theoretical calculations that one can gain insight into the internal dynamics. Here, we report on a combination of structural (relativistic ultrafast electron diffraction, or UED) and spectroscopic (time-resolved photoelectron spectroscopy, or TRPES) measurements to follow the coupled electronic and nuclear dynamics involved in the internal conversion and photodissociation of the polyatomic molecule, diiodomethane $\left(\mathrm{CH}_{2} \mathrm{I}_{2}\right)$. While UED directly probes the 3D nuclear dynamics, TRPES only serves as an indirect probe of nuclear dynamics via Franck-Condon factors, but it is sensitive to electronic energies and configurations, via Koopmans' correlations and photoelectron angular distributions. These two measurements are interpreted with trajectory surface hopping calculations, which are capable of simulating the observables for both measurements from the same dynamics calculations. The measurements highlight the nonlocal dynamics captured by different groups of trajectories in the calculations. For the first time, both UED and TRPES are combined with theory capable of calculating the observables in both cases, yielding a direct view of the structural and nonadiabatic dynamics involved.
\end{abstract}

DOI: 10.1103/PhysRevX.10.021016

Subject Areas: Atomic and Molecular Physics, Chemical Physics, Physical Chemistry

\footnotetext{
*philipp.marquetand@univie.ac.at

†wangxj@slac.stanford.edu

thomas.weinacht@stonybrook.edu
}

Published by the American Physical Society under the terms of the Creative Commons Attribution 4.0 International license. Further distribution of this work must maintain attribution to the author(s) and the published article's title, journal citation, and DOI. 


\section{INTRODUCTION}

Following the electronic and structural dynamics of molecules on femtosecond timescales is key to understanding many fundamental processes in physics, chemistry, and biology, such as the photoprotection of DNA [1], the basic steps involved in vision [2,3], and how energy and charge transfer facilitates light harvesting [4,5] and energy conversion in photoabsorption [6]. While experimental measurements of these dynamics have provided considerable insight over the past few decades [7], it is not generally possible to convert the experimental measurements directly into time-dependent structures or charge distributions. Timedependent calculations have evolved to produce very detailed pictures of molecular dynamics, but they require many approximations and thus must be benchmarked against measurements in order to ensure accuracy and relevance. It is important that these simulations can be directly compared (without adjustable parameters) to time-resolved experimental data, as has been done, e.g., with quantum dynamics simulations [8-12], trajectory surface hopping calculations [13-15], ab initio multiple spawning (AIMS) [16-21], and many more approaches. While a given observable may apply certain constraints to a calculation, many ambiguities will remain. It is useful, therefore, to apply more than one observable to a given dynamics, thus more tightly constraining the theoretical simulations and yielding a more accurate picture of the dynamics involved. Here, we compare two complementary measurements, ultrafast electron diffraction (UED) [22] and time-resolved photoelectron spectroscopy (TRPES) [23], with trajectory surface hopping calculations of UV excited dynamics in $\mathrm{CH}_{2} \mathrm{I}_{2}$.

Each experimental approach provides a useful perspective but rarely provides a complete picture of the evolving chemical dynamics, particularly in polyatomic systems that are inherently multidimensional. For example, optical spectroscopies, such as transient absorption [24-28], can provide high time resolution with a compact apparatus but require detailed knowledge of the potential energy surfaces (electronic energies as a function of nuclear coordinates) and transition dipole moments along the reaction coordinate in order to be interpreted. Time-resolved ionization spectroscopies [23,29-36] offer the advantage over optical spectroscopies that it is always possible to ionize, regardless of the character of the excited-state, and if one measures the energy of the photoelectrons as a function of pump-probe delay, then one can extract information about the distribution of energy within the molecule as a function of time. UED [37-43] holds the promise of providing direct structural information as a function of time, but it suffers from orientational averaging over the sample, repulsion between the electrons in a short pulse, and the group velocity mismatch between electrons and light. Ultrafast $\mathrm{x}$-ray diffraction overcomes the last two disadvantages of electron diffraction [44-47], but it suffers from low scattering cross sections and requires a large number of photons in the probe pulse - typically only available at free electron laser (FEL) light sources [48-51]. One way to mitigate the electron repulsion and group velocity mismatch with UED is to work with relativistic electrons, which spread much less due to Coulomb repulsion and travel close to the speed of light, leading to much better group velocity matching with the pump pulse over the sample volume and thus higher time resolution [20,21,52-54].

Here, we probe the time-dependent electronic structure (energies and configurations) and nuclear configurations (relative positions of the atoms) of the molecule $\mathrm{CH}_{2} \mathrm{I}_{2}$ using TRPES and relativistic UED following UV photoexcitation [55]. While relativistic UED can directly capture molecular structure (nuclear wave functions or positions) with about 100 fs time and sub-Angström spatial resolution, it does not

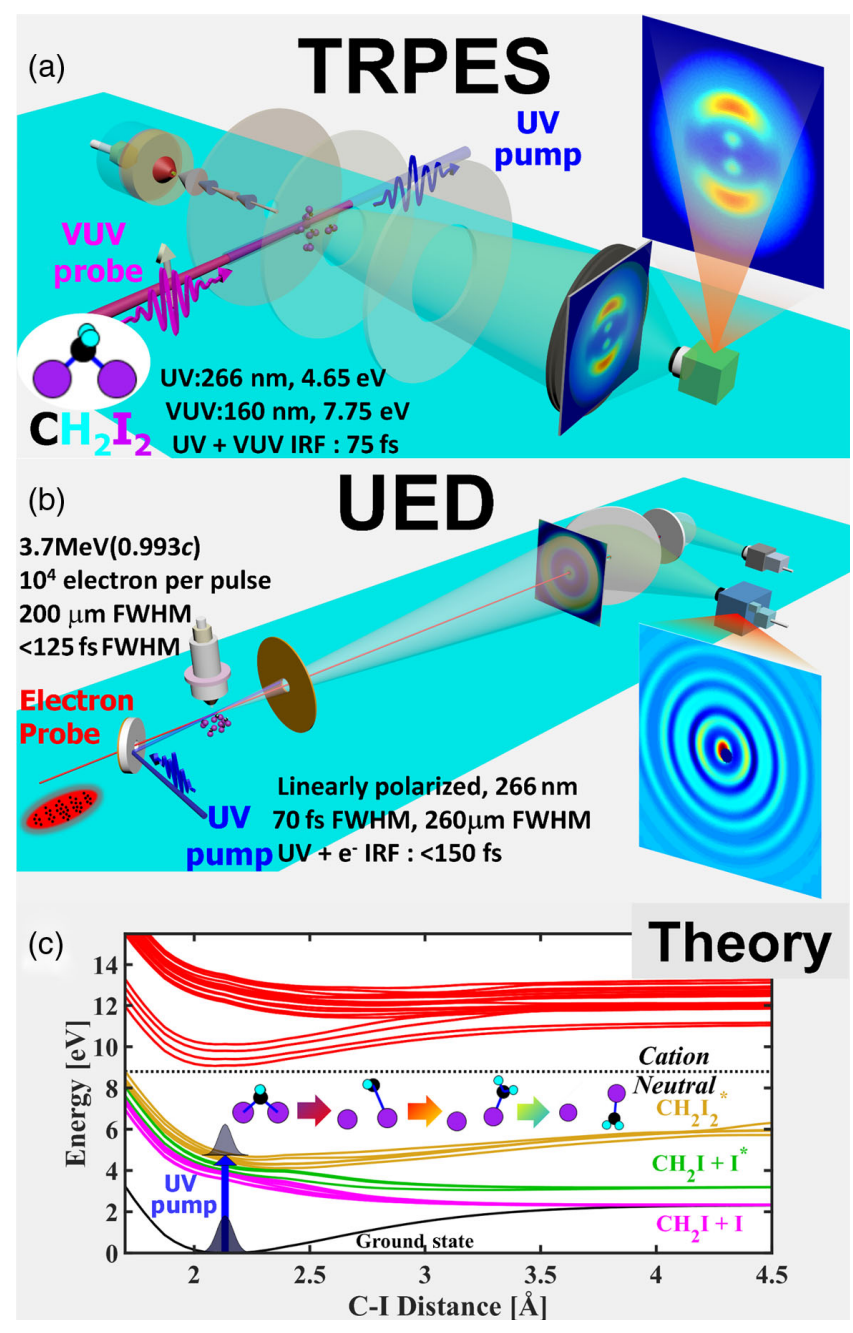

FIG. 1. Schematic diagrams illustrating the experimental methods and calculations to follow the photoinduced excited-state dynamics of $\mathrm{CH}_{2} \mathrm{I}_{2}$. (a) A schematic diagram of the time-resolved photoelectron spectroscopy experiment. A cartoon of the $\mathrm{CH}_{2} \mathrm{I}_{2}$ molecule is shown in the bottom-left corner. (b) A schematic diagram of the relativistic ultrafast electron diffraction experiment. Two cameras are responsible for recording images of scattered and unscattered electrons. (c) A cartoon of potential energy curves together with structural changes of $\mathrm{CH}_{2} \mathrm{I}_{2}$ after UV excitation. A black dotted line separates the neutral and cation states. 
provide information about the electronic state (electronic wave functions or positions) or energy of the molecule. TRPES can directly measure the electronic energy and state character with sub 100 fs time and millielectron volt (meV) energy resolution, but it does not provide direct information about structure. Furthermore, the nonadiabatic coupling between electrons and nuclei underlying many fundamental light driven processes in nature (photosynthesis, photoprotection of DNA, vision, etc.) is best unveiled when one has direct access to both electronic and nuclear degrees of freedom. Thus, in order to form a complete picture of time-dependent molecular transformation, including both energy and structure as well as nonadiabatic coupling, one needs to combine the information from the two approaches. This method has never been used before the present work.

Figures 1(a) and 1(b) illustrate the experimental approaches. The measurements are compared with trajectory surface hopping calculations [56], which simulate the observables for both measurements based on the same time-dependent calculations. Figure 1(c) shows the $\mathrm{CH}_{2} \mathrm{I}_{2}$ electronic structure along the C-I dissociation coordinate, and a series of cartoon snapshots illustrate the C-I dissociation dynamics after UV excitation. The combination of structural and spectroscopic probing in conjunction with the calculations provides new views of the dynamics, which involve nonadiabatically coupled electronic-nuclear dynamics, complex structural changes, and nonlocal evolution of the molecular wave function (bifurcation and spreading).

The choice of $\mathrm{CH}_{2} \mathrm{I}_{2}$ for this comparative study was motivated by a number of factors. The excited-state dynamics are sufficiently rich that calculations must be compared with experimental measurements to verify their validity. $\mathrm{CH}_{2} \mathrm{I}_{2}$ exhibits a number of features that are intrinsically interesting and representative of many polyatomic systemsnonadiabatic coupling between electronic states, spin-orbit coupling, dissociation, and concerted motion along multiple degrees of freedom. The presence of heavy I atoms makes it well suited for diffractive measurements since the scattering cross section is large, providing a high signal-to-noise ratio (SNR). Finally, while the photoproducts have been studied in some detail with nanosecond laser work [57-67], the femtosecond dynamics following deep UV photoexcitation have not been studied in such detail.

\section{EXPERIMENTAL METHODS}

The experimental measurements were carried out in two different laboratories, but we made use of the same excitation scheme. Both the TRPES and UED measurements were made on an ensemble of $\mathrm{CH}_{2} \mathrm{I}_{2}$ molecules excited with linearly polarized pump pulses in the deep UV, having a central wavelength around $266 \mathrm{~nm}$ (photon energy of $4.65 \mathrm{eV}$ ) and a pulse duration of about 50-60 fs.

\section{A. TRPES experiment}

The TRPES experiments utilized a standard pump-probe spectroscopy scheme [68]. A seeded supersonic beam injected $\mathrm{CH}_{2} \mathrm{I}_{2}$ molecules through a pulsed valve into the interaction region of an electron velocity map imaging (VMI) spectrometer [69], where they interacted with the fs UV-pump and VUV-probe pulses. The laser pulses were weakly focused using $f / 100$ spherical reflective optics, yielding a UV pump laser intensity of about $5 \times 10^{11} \mathrm{~W} / \mathrm{cm}^{2}$. Measurements performed with pump-pulse intensities between 0.3 and $1.5 \times$ $10^{12} \mathrm{~W} / \mathrm{cm}^{2}$ yielded similar results. The VUV probe laser intensity was more than an order of magnitude weaker. The photoelectrons generated by the pump and probe pulses were measured with a VMI spectrometer for each pump-probe delay, $\Delta \tau$. VUV pulses $(160 \mathrm{~nm}, 7.75 \mathrm{eV})$ were used as a probe of the dynamics, ionizing the excited molecules at variable delay after initial UV excitation [70,71]. Figure 1(a) shows a schematic diagram of the TRPES experiment. A cartoon of the $\mathrm{CH}_{2} \mathrm{I}_{2}$ molecule is shown in the bottom-left corner.

The photon energy of the probe was chosen to maximize the observation window for the excited-state dynamics while avoiding ionization of the ground state $[23,72]$. The impulse response function (IRF) of the system was determined to be 75 fs full width at half maximum (FWHM) via nonresonant two-photon ionization of Xe. This reuslt allows us to follow the fast dynamics at early pump-probe delay times. The highest energy photoelectrons observed corresponded to those expected of a one-photon pump, onephoton probe measurement, thus ruling out the possibility of two-photon pump processes, which was in line with the low pump fluence used for these measurements. With this weakfield (single-photon) interaction as a probe, simulation of the TRPES spectrum is possible, allowing for direct comparison with experiment. Further information regarding the experimental apparatus can be found in Ref. [73].

\section{B. UED experiment}

The UED experiment discussed in this work utilized the ultrafast relativistic mega-electron-volt $(\mathrm{MeV})$ electron beam line at the Stanford Linear Accelerator Center [55,7477]. $\mathrm{CH}_{2} \mathrm{I}_{2}$ molecules were injected with a pulsed nozzle into the reaction chamber, where they interacted with the UV-pump pulse. In order to achieve a sufficient signal-tonoise ratio for the interpretation of the measurements, we worked at pump intensities of about $10^{12} \mathrm{~W} / \mathrm{cm}^{2}$, which led to some two-photon absorption from the pump pulse. Based on an analysis of the angle-dependent diffraction signal, a scan of the pump-pulse intensity, and the strength of different features in the pair distribution function, we estimate the two-photon absorption to be about $10 \%$ of the one-photon absorption. The details are provided in the Appendix C. In contrast, with the TPRES measurements, the probe pulse consisted of relativistic electrons, which were elastically scattered by the molecules and projected onto a phosphor screen in the far field. The time-dependent molecular structure was thus imprinted onto a series of diffraction patterns taken for each pump-probe delay. These patterns were recorded by a charged-coupled device (CCD) 
camera. The experimental layout used in this work is shown in Fig. 1(b).

The kinetic energy of electrons in the beam line was measured to be $3.7 \mathrm{MeV}\left(\lambda_{e} \sim 0.003 \AA\right)$, providing us with excellent spatial and temporal resolution [75]. At this energy, the electrons are relativistic, leading to a negligible velocity mismatch between the laser and electron pulses and very limited Coulomb broadening. This case allows us to achieve an overall instrumental IRF of under $150 \mathrm{fs}$ FWHM in the system [20]. The largest momentum transfer value on the detector is $S_{\max }=11.5 \AA^{-1}$, which gives a spatial resolution of $\delta=0.54 \AA$ [53].

\section{CALCULATION}

\section{A. Trajectory surface hopping calculation}

In order to interpret the experimental results and produce a detailed picture of the energetic and structural evolution of the molecule following photoexcitation, we carried out trajectory surface hopping calculations of the excited-state molecular dynamics. We used the results of these calculations to produce the same observables obtained in the UED and TRPES measurements. In order to carry out the excited-state dynamics simulations, we used the $a b$ initio molecular dynamics program SHARC (surface hopping including arbitrary couplings) [78,79], interfaced with MOLCAS 8.0 [80]. The electronic structure calculations were performed with MS-CASPT2(12,8)/ano-rcc-vdzp (multistate complete active space perturbation theory second order) based on SA(5/4)-CASSCF(12,8) (complete active space self-consistent field with 12 electrons in 8 orbitals), where the state averaging included five singlet and four triplet states for the valence states excited with one photon absorption from the pump pulse. Additionally, the dynamics in a high-lying Rydberg state (accessible via the absorption of two pump photons) was calculated by approximating the potential in terms of the ground electronic state of the molecular cation. The Rydberg state $a b$ initio molecular dynamics simulations were carried out with SHARC based on CASPT2(11,8)/ano-rcc-vdzp for the doublet ground state of the cation.

\section{B. Calculation of observables}

In order to calculate the TRPES, the ionization probability along the time-resolved trajectories was obtained in an approximate manner from calculations of the Dyson orbital norms [81], using our wave-function overlap (WFOverlap) code [82] in a postprocessing step. The necessary wave functions for the neutral and ionized molecules were obtained at steps of $2.5 \mathrm{fs}$ along the precomputed trajectories from MS-CASPT2 $(12,8) /$ ano-rcc-vdzp or MS-CASPT2 $(11,8)$ /ano-rcc-vdzp calculations, including altogether five singlets, nine doublets, four triplets, and four quartets, as well as all possible spin-orbit couplings (SOCs).
In order to calculate the time-resolved pair distribution function (PDF), we computed the electron diffraction pattern for the time-dependent molecular geometries extracted from the surface hopping calculation given that each trajectory included all of the atomic positions as a function of delay. The diffraction patterns were simulated within the independent atom model for each molecular geometry, with the elastic scattering amplitudes for C, I, and $\mathrm{H}$ atoms calculated using the Dirac partial-wave method (ELSEPA) [83]. Experimental conditions were applied to the diffraction pattern simulation in order to compare with the measurements. Details are provided in Appendix B 3 .

\section{RESULTS}

We first present the TRPES results, which have a lower information density than the UED results but still provide important insights. Figure 2 shows the measured and calculated photoelectron spectra as a function of pump-probe delay. In the molecular ground state, $\mathrm{CH}_{2} \mathrm{I}_{2}$ absorbs strongly at wavelengths corresponding to both our pump and probe laser pulses, which means that near time zero, when the pump and probe pulses overlap, the signal has contributions from both UV and VUV driven dynamics. Figure 2(a) shows the experimental data [73]. In order to highlight the UV driven dynamics, we multiply the signal at positive time delays outside the temporal overlap of pump and probe pulses by a factor of 4 since we estimate that the VUV absorption cross section is about 4 times that of the UV absorption cross section [66]. Figure 2(b) presents the results of the surface hopping calculations, which provide the calculated photoelectron spectrum versus pump-probe delay.

One can see that the calculations and measurements agree on the presence of a broad peak near zero time delay, as well as a longer tail at low photoelectron energies.

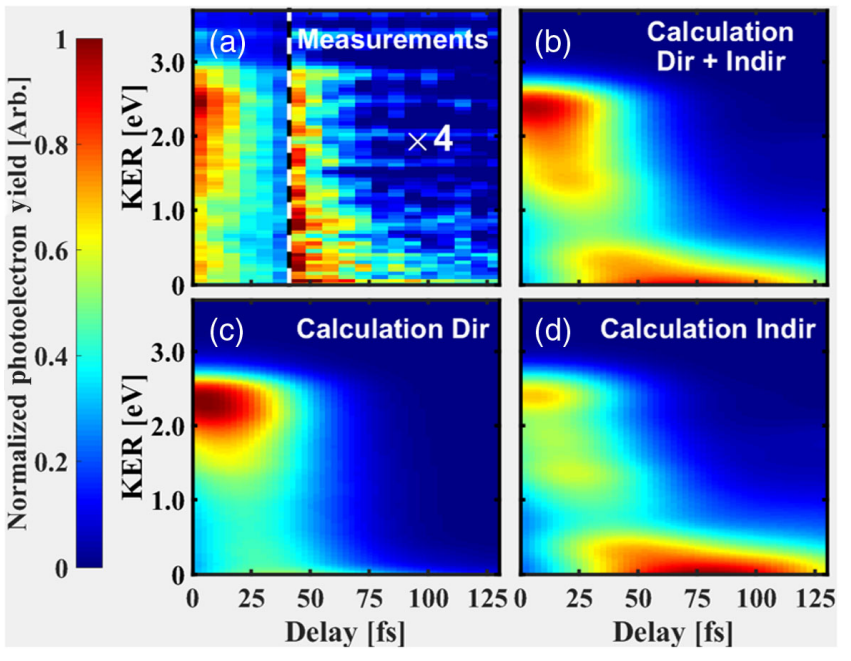

FIG. 2. Measured and simulated time-resolved photoelectron spectra. (a) TRPES from experimental measurement. (b) Simulated TRPES including all trajectories. (c) Simulated TRPES for direct (Dir) dissociation trajectories. (d) Simulated TRPES for indirect (InDir) dissociation trajectories. 
The broad peak at zero time delay extends over a broad range of energies up to $2.5 \mathrm{eV}$ and corresponds to ionization near the Franck-Condon (FC) region on the excited-state surface to several low-lying states of the cation. As there is rapid motion of the wave packet leaving the $\mathrm{FC}$ region, and the cationic states are close to each other [see Fig. 1(c)], one cannot resolve the ionization to different cationic states, which can be seen in examining the calculations. In both measured and calculated spectra, the higher energy peak extending up to $2.5 \mathrm{eV}$ lasts for $50 \mathrm{fs}$ and is followed by a low-energy tail persisting out to beyond 100 fs. This behavior is consistent with the excited-state wave packet moving rapidly to regions with larger vertical IPs, which is mapped to the decrease of the observed KER. The presence of a long tail at low energies is consistent with some population remaining on the $\mathrm{CH}_{2} \mathrm{I}_{2}^{*}$ potentials shown in Fig. 1 for times out beyond 100 fs. In order to explore this idea, the trajectories underlying the calculated TRPES (Fig. 2) can be analyzed in detail.

In performing a more detailed analysis of the TRPES, a natural question is whether different trajectories are responsible for the different features in the photoelectron spectrum. In order to address this question, our previous work looked for and found a correlation between trajectories that led to symmetric C-I bond stretching and produced a low-energy tail in the photoelectron spectrum versus trajectories that featured asymmetric C-I stretching and did not contain a low-energy tail [73]. We thus sorted the trajectories into two groups based on whether they displayed symmetric or asymmetric stretching of the C-I bonds. As the asymmetric stretch trajectories involve rapid internal conversion and dissociation of an iodine atom, we denote these as direct dissociation trajectories. In contrast, the symmetric stretching trajectories involve some motion on the potential of the initial states followed by slower internal conversion and dissociation on longer timescales. We thus label these indirect dissociation trajectories. In Fig. 2, the TRPES for these two groups of trajectories are shown in panels (c) and (d). Panel (c) shows the TRPES for the direct dissociation trajectories and panel (d) shows the TRPES vs delay for the indirect dissociation trajectories. It is clear that the low-energy tail in the TRPES can be associated with the indirect dissociation trajectories. This case motivates an interpretation of the UED measurements in terms of these two groups of trajectories. We also note that Fig. 14 in Appendix B contains a comparison between the measurements and calculations with and without surface hopping. This comparison illustrates how the internal conversion can be seen in the TRPES measurements.

The UED measurements yield a two-dimensional projection of the three-dimensional far-field diffraction pattern for each pump-probe delay. This far-field diffraction pattern represents the momentum-space interference pattern of the scattered electrons. The diffraction difference was first obtained by subtracting the diffraction pattern for negative time delays (averaged over four delays) [84]. The difference pattern was then Fourier transformed to produce a twodimensional position-dependent distribution. Given the cylindrical symmetry of the photoexcited molecular ensemble (symmetry around the linear polarization vector of the

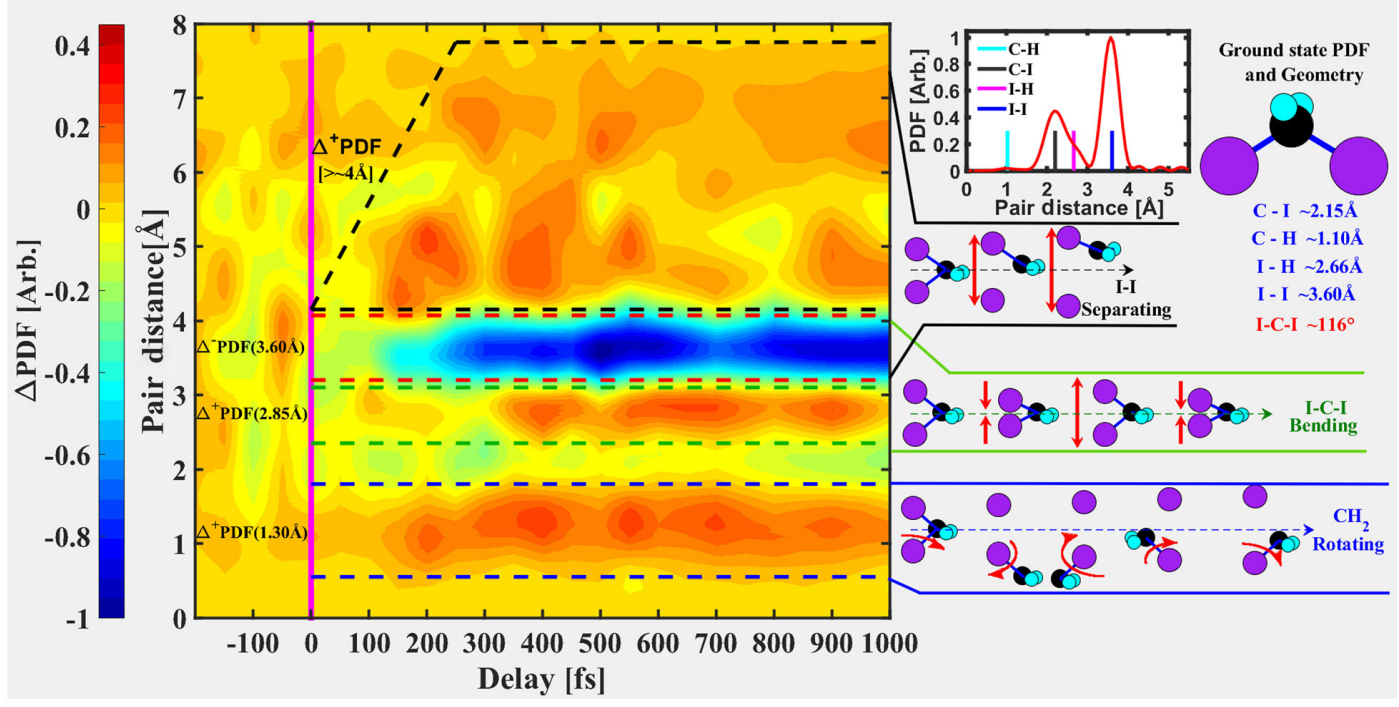

FIG. 3. Measured $\triangle \mathrm{PDF}$ as a function of pump-probe delay. A vertical magenta-colored line marks zero pump-probe delay. The data are normalized by the largest value of $\triangle \mathrm{PDF}$. Four regions corresponding to specific molecular motions are highlighted with dashed lines in different colors. Snapshots on the right side of the graph illustrate the different types of motion highlighted in the regions bounded by the dashed lines to the left. A cartoon picture on the top right of the graph shows the ground-state equilibrium geometry of $\mathrm{CH}_{2} \mathrm{I}_{2}$, with black, purple, and cyan colored balls representing the carbon, iodine, and hydrogen atoms, respectively. The atomic pair distances (blue) and I-C-I angle (red) are labeled underneath. The corresponding ground-state PDF and atomic pair distances are plotted adjacently. 
pump laser pulse), we were able to perform an inverse Abel transform of the measured spatial distribution function to yield the three-dimensional pair distribution function for each time delay. Given the high-dimensional nature of this information (three spatial dimensions + time), in order to provide a first overview of the measurements, we integrated over both polar (with respect to the pump-pulse polarization axis) and azimuthal angles to form a two-dimensional distribution that shows the scattering intensity as a function of interatomic distance and pump-probe delay. This distribution is shown in Fig. 3. However, analyzing the polar angle dependence of the measurements can yield valuable information, which we make use of later in Fig. 4, as well as in an analysis of multiphoton absorption from the pump pulse discussed in Appendix C.

Figure 3 shows the scattered intensity as a function of interatomic distance - the PDF - and pump-probe delay for $\mathrm{CH}_{2} \mathrm{I}_{2}$ (ground-state equilibrium geometry shown in the top-left corner) from experimental measurements. The PDF for negative time delays is subtracted from the PDF for positive delays, producing the $\triangle \mathrm{PDF}$. This process allows

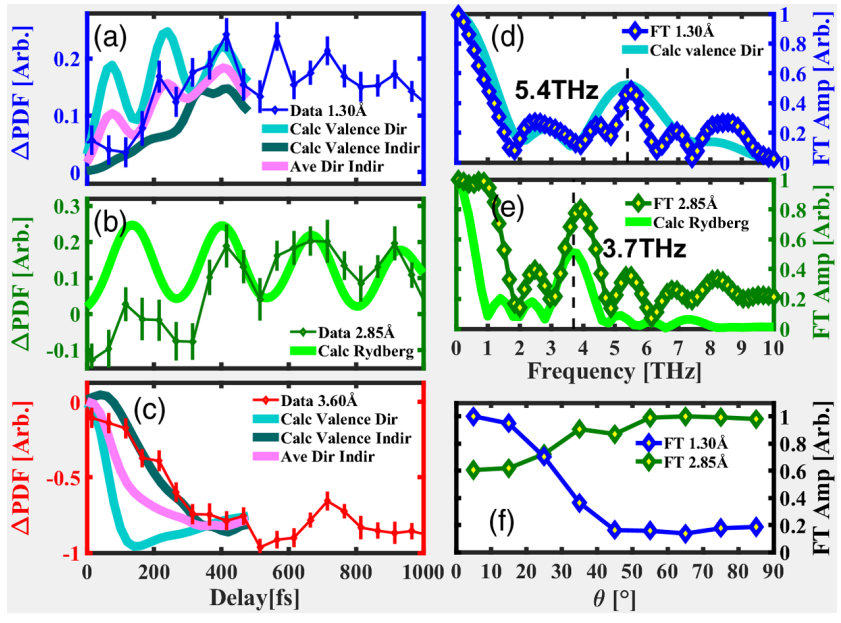

FIG. 4. Time-resolved $\triangle \mathrm{PDF}$ at specific pair distances and their Fourier transforms for both experiment and simulation. (a) Experimental and simulated time-resolved $\triangle \mathrm{PDF}$ around $1.30 \AA$. The simulated $\triangle \mathrm{PDF}$ are calculated from the groups of both direct (Dir) and indirect (InDir) dissociative trajectories. (b) Experimental and simulated time-resolved $\Delta \mathrm{PDF}$ around $2.85 \AA$. The simulation is from the group of Rydberg state trajectories. (c) Experimental and simulated time-resolved $\triangle \mathrm{PDF}$ around $3.60 \AA$. The simulations are from both direct and indirect dissociative trajectories. The pink curves in panels (a) and (c) are for an equally weighted average of direct and indirect trajectories. Appendix B 4 contains a comparison of the data and simulation with different mixtures of direct and indirect trajectories. (d) Fourier analysis of $\triangle \mathrm{PDF}$ at $1.30 \AA$ from experimental data and simulation of direct dissociation trajectories [panel (a)] for a polar angle of $0^{\circ}$. (e) Fourier analysis of $\triangle \mathrm{PDF}$ at $2.85 \AA$ from experimental data and Rydberg state trajectories [panel (b)] for a polar angle of $70^{\circ}$. (f) Angle-dependent Fourier analysis of the $\Delta \mathrm{PDF}$ at $1.30 \AA$ and $2.85 \AA$. Note that $\theta$ is the angle with respect to the laser polarization direction. us to focus on the changes in the PDF due to excited-state dynamics and to subtract the ground-state contributions as well as those from individual atoms, focusing on the molecular or interference contributions arising from the excited-state(s) of the molecule. Blue coloring indicates loss, and red indicates gain compared with the unexcited molecules. A solid vertical magenta-colored line indicates zero time delay-i.e., where the pump laser and probe electron bunch are overlapped in time. The delay-dependent PDF shows more structure (features that vary with delay and pair distance) than the TRPES results, providing a more detailed view of the dynamics than the delay-dependent photoelectron spectrum in these measurements.

In order to interpret the time-resolved $\triangle \mathrm{PDF}$, we calculated the ground-state pair distribution function and plotted the geometry with relevant atomic pair distances and angles in Fig. 3. The ground-state PDF shows two main peaks at $2.15 \AA$ and $3.60 \AA$, arising from the C-I and I-I pairs, respectively. Because of the relatively low electron scattering cross section of $\mathrm{H}$ atoms compared with $\mathrm{C}$ and $\mathrm{I}$ atoms, the contributions related to the $\mathrm{H}$ atoms are negligible. Compared with the ground-state PDF, one can see that in the measured $\triangle \mathrm{PDF}$, there are several characteristic atomic pair distances that are of interest for the dynamics we follow, and we highlight a number of features in the data at these pair distances that can be interpreted in terms of specific types of motion. They are illustrated by the cartoon diagrams on the right-hand side of the figure. The top cartoon illustrates I-I separation with increasing time delay. As the I atoms have the largest scattering cross section, the I-I contribution to the changing PDF is the largest. The middle cartoon illustrates I-C-I bending that takes place in an electronic state accessed by two-photon absorption from the pump pulse, and the lowest cartoon illustrates motion of the $\mathrm{CH}_{2}$ as one of the C-I bonds is broken. These dynamics are captured by the UED measurements at different pair distances bounded by pairs of dashed lines in the main figure.

There is a deep blue stripe centered at $3.60 \AA$ Andicating a large decrease in the probability of finding I atoms separated by their equilibrium distance. Together with the decrease of the PDF at $3.60 \AA$, the signal shows modulated increases for distances greater than $4 \AA$ and two narrower regions centered at 1.30 and $2.85 \AA$. The increase in the PDF for distances greater than $4 \AA$ is expected and has contributions from both C-I and I-I pairs, with both arising directly from the dissociation of the molecule in the excited-states. Given the scattering cross sections for C and I atoms, it is dominated by I-I contributions, as illustrated by the cartoon on the right-hand side of the figure. While the modulations for distances greater than $4 \AA$ have no obvious periodicity, the modulations in the PDF at $1.30 \AA$ and $2.85 \AA$ do. These modulations are surprising and highlight two different kinds of motion, which are illustrated to the right-hand side of the figure and discussed below. 


\section{DISCUSSION}

In order to interpret the experimentally measured PDF and validate the calculations, we compared the measured and calculated PDF at the distances bordered by the dashed lines in Fig. 3. Motivated by the analysis of the TRPES measurements, we compared the UED measurements with calculations for the two different groups of trajectories (direct and indirect dissociation), as well as trajectories for dynamics in high-lying Rydberg states in order to account for molecular dynamics driven by two-photon absorption from the pump pulse.

The modulations in the PDF at $2.85 \AA$ are not found in our calculations of the valence state dynamics (i.e., states excited by one-photon absorption), but the periodicity of the modulations corresponds to I-C-I bending motion in high-lying Rydberg states or low-lying states of the cation $[85,86]$. Since these states can be accessed by two-photon absorption from the pump, we performed calculations of the dynamics in high-lying Rydberg states, modeled by considering the ground state of the molecular cation, for which the potential energy surface is roughly parallel to the Rydberg states excited by two-photon absorption [34]. The shapes of the potential energy surfaces for the Rydberg and ionic states are very similar since they are both determined by the removal of a HOMO (iodine lone pair) electron, which leads to displacement along the I-C-I bending coordinate.

The modulations in the $\triangle \mathrm{PDF}$ centered at $1.30 \AA$ are also present in our calculations as shown in Fig. 4, but they do not correspond to periodic modulation of any atom pair distance. Rather, they are due to rotation of the $\mathrm{CH}_{2}$ group after the bond breaks between the $\mathrm{C}$ and one of the two I atoms. The rotations lead to a periodic oscillation in the projection of the remaining (unbroken) C-I bond distance onto the plane of the detector with a period equal to half the rotational period since the pump laser only aligns the molecules but does not orient them. The I-C-I angle as a function of time for direct dissociation trajectories is shown in Appendix B. To our knowledge, the modulations we observe here represent the first direct time-resolved observation of fragment rotation in photodissociation.

Figure 4 shows the time evolution of $\triangle \mathrm{PDF}$ at several pair distances [panels (a)-(c)] and their Fourier transforms [panels (d) and (e)] for both UED measurements and simulation. Panels (d) and (e) show the absolute value of the Fourier-transformed data given in panels (a) and (b), respectively. The thick dashed lines represent simulation results, while the thin lines with diamond markers represent the experimental measurements. In panel (a), both the measurement and simulation show periodic oscillations at a frequency of $5.4 \mathrm{THz}$. In Fig. 4(b), both the data and simulation show a periodic oscillation at a frequency of 3.7 THz. In both panels (a) and (b), the first period of oscillation in the experimental data has a lower signal level than the calculations, due to the subtraction of the signal for negative time delays with finite pump and probe durations. Figure 4(c) shows the calculated and measured $\triangle \mathrm{PDF}$ at $3.60 \AA$ as a function of pump-probe delay. There are no modulations but rather a rapid monotonic decrease in the signal. The analysis here makes use of the angle dependence of the measured and calculated $\triangle$ PDFs. The results shown in panels (a)-(c) used the angular-averaged $\triangle \mathrm{PDF}$ values. The results in panel (d) used $\triangle \mathrm{PDF}$ values along a polar angle of $0^{\circ}$, while the results in panel (e) used $\triangle \mathrm{PDF}$ values along a polar angle of $70^{\circ}$. Panel (f) highlights the angle dependence of the signal by showing the heights of the peaks in panels (d) and (e) as a function of polar angle. A detailed analysis of the polar angle dependence of the PDF is given in Appendix A 2.

The data persist out to $1 \mathrm{ps}$, while the simulations in panels (a) and (c) only last to 500 fs because the dissociative trajectories tend to crash as a result of an insufficient active space. The agreement between the measurements and calculations shown in panels (a), (c), and (d) of Fig. 4 indicates that the structural changes associated with the groups of direct and indirect dissociation trajectories in the lower-lying electronic states (states $8-17$ in Fig. 5) are captured by and directly reflected in the UED measurements. The agreement between the measurements and calculations shown in panels (b) and (e) of Fig. 4 indicates that the dynamics in the Rydberg states are also captured by the UED measurements and can be separated from the dynamics in the lower-lying states [20].

Both the TRPES and UED measurements show features that can be associated with the two different groups of trajectories-directly dissociative and indirectly dissociative. These features highlight both the nonlocal nature of the photoexcited wave-packet dynamics (bifurcation of the nuclear wave packet and the exploration of multiple regions of coordinate space), and the nonadiabatic dynamics involved. It is interesting to note, however, that trajectories within a given group show limited dispersion, facilitating the formation of two groups of trajectories.

As the differences between trajectories in each group of direct or indirect dissociation are much smaller than the differences between the two groups (see Appendix B 2 for a detailed analysis), we choose one trajectory from each group to be a representative and explore the details from both spectroscopic and structural perspectives. Figure 5 provides both a spectroscopic and structural view of representative direct and indirect dissociation trajectories. Panel (a) plots the relevant potential energy curves along the C-I dissociation coordinate and shows cartoon snapshots of the wave packet for both direct and indirect dissociation trajectories. Note that, for simplicity, we grouped the excited-states shown in panel (c) of Fig. 1 into three bands and plotted them as a function of C-I bond distance. The wave packet associated with the direct dissociation trajectories moves out to large C-I distances rapidly after internal conversion from the initially photoexcited states (13-17) to the 

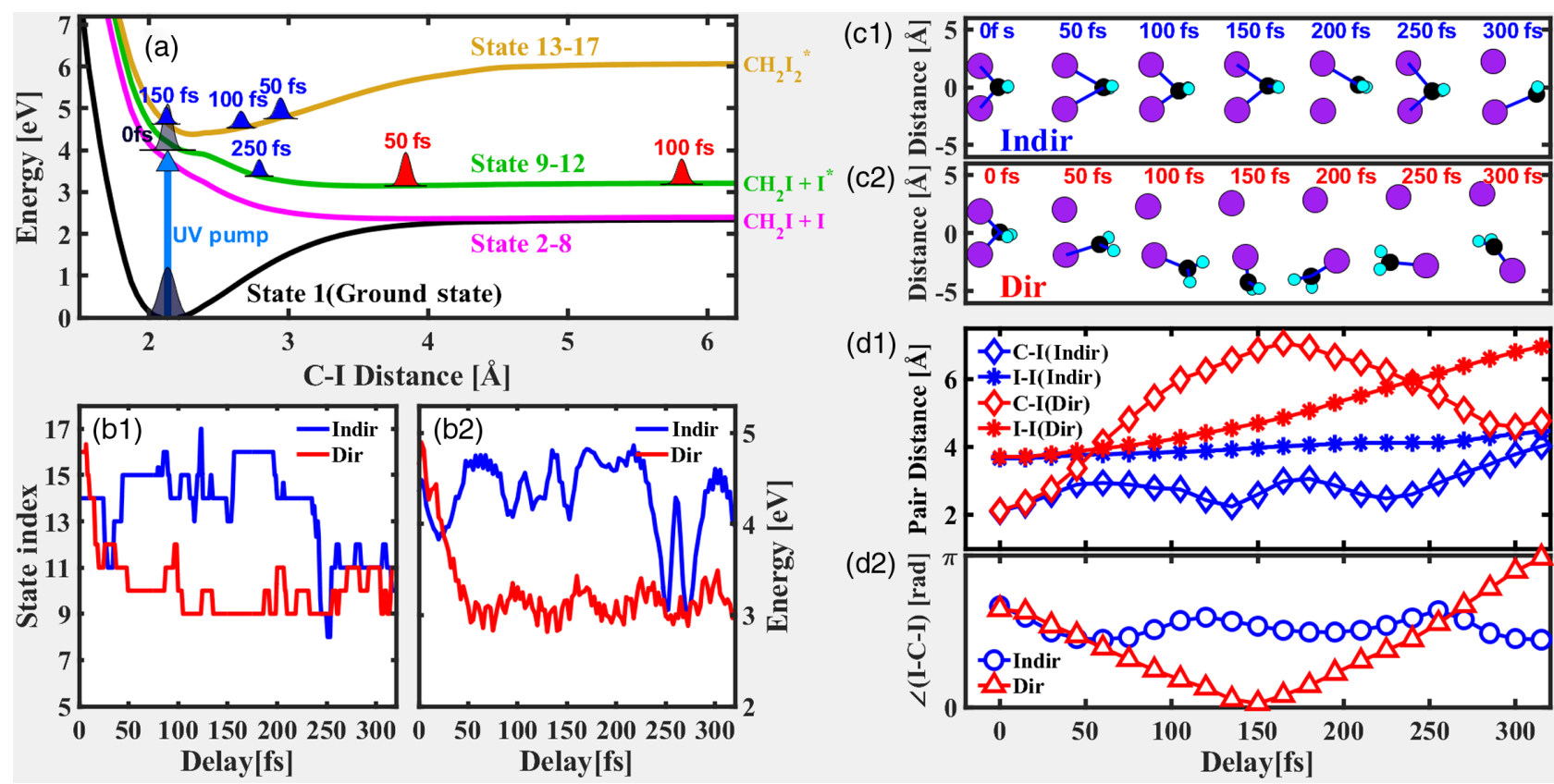

FIG. 5. Spectroscopic and structural views of $\mathrm{CH}_{2} \mathrm{I}_{2}$ dissociation dynamics. (a) Potential energy curves and cartoon snapshots of the wave packet at different time delays for indirect dissociation (blue) and direct dissociation (red) trajectories. (b1) Time-evolved electronic state index for indirect (blue) and direct (red) dissociation trajectories. (b2) Time-evolved potential energy for indirect (blue) and direct (red) dissociation trajectories. (c1) Snapshots of the molecular structure at different time delays for an indirect dissociation trajectory. (c2) Snapshots of the molecular structure at different time delays for a direct dissociation trajectory. The frames show 2D projections of the 3D molecule onto the I-C-I plane. (d1) Time evolution of pair distances for indirect (blue) and direct (red) dissociation trajectories. (d2) Time evolution of the I-C-I angle from the indirect and direct dissociation trajectories.

dissociative states (9-12), while the wave packet associated with the indirect trajectories remains bound in states 13-17 for about 200 fs and then eventually internally converts, followed by dissociation. Panels (b1) and (b2) show the electronic state index as well as the potential energy as a function of delay time after the UV pump pulse. The two trajectories display very different behavior. The difference between the internal conversion rates and energy loss for the two different trajectories is reflected in panels (b1) and (b2).

Panels (c1) and (c2) of Fig. 5 show snapshots of timeresolved geometry changes for the chosen trajectories. In panel (c1), we show the structure as a function of time for the indirect dissociation trajectory. One can see that there are relatively small changes, with the initial motion dominated by symmetric stretching of the two C-I bonds. However, for the direct dissociation trajectory shown in panel (c2), one of the C-I bonds breaks quickly, leading to rotation of the $\mathrm{CH}_{2}$ group around the other I atom. The slow dissociation dynamics for the indirect trajectories is directly captured by the UED measurements in the I-I depletion shown in panel (c) of Fig. 4, while the rapid $\mathrm{CH}_{2}$ rotation in the direct dissociation trajectory is directly captured by the UED measurements in the modulation of the PDF near $1.30 \AA$, as shown in panels (a) and (d) of Fig. 4. The corresponding C-I and I-I distances can be viewed in panel (d1), while the I-C-I angle is shown in panel (d2). Note that the $\mathrm{CH}_{2}$ rotation frequency is about
2.7 THz, corresponding to a period of $370 \mathrm{fs}$, which is half of the period shown in panel (d) of Fig. 4. This result is due to the fact that the projection of the $\mathrm{C}$-I bond that remains in the rotating $\mathrm{CH}_{2} \mathrm{I}$ fragment has roughly the same value twice per rotational period. This direct view of the threedimensional dynamics, including wave-packet bifurcation, rotation, and dissociation, provides a very clear and detailed picture of the dynamics as well as a very compelling verification of the calculations.

Before concluding, in light of the analysis and discussion above, we compare the information available from the two measurement approaches. TRPES provides direct information on the time-dependent electronic energy of the molecule, with indirect information on electronic and nuclear configurations, while UED provides direct information on nuclear configurations. TRPES measurements can rather easily be carried out in the limit of one-photon absorption from the pump pulse, with built-in verification via the energy of the photoelectrons, while UED requires higher pump-pulse fluences and thus tends to contain contributions from dynamics driven by multiphoton absorption from the pump pulse, which need to be considered in the analysis. While the time resolution of the two approaches is similar, it is easier to characterize the IRF for TRPES via cooperative nonresonant $1+1$ ' photon absorption. The rate at which the UED observable changes depends primarily on how fast the structure changes with 
time ( $\left(d \mathbf{R}_{i} / d t\right)$, where $\mathbf{R}_{i}$ represents the position of the ith nucleus), whereas the TRPES observable depends on both the rate of structural change with time and how rapidly the energy changes with structure-i.e., $\left(d \mathbf{R}_{i} / d t\right)$ and $\left[d V\left(\mathbf{R}_{i}\right) /\left(d \mathbf{R}_{i}\right)\right]$. This case leads to the TRPES observable changing more rapidly than UED for some time delays (e.g., less than $100 \mathrm{fs}$ ) and less rapidly than UED for others (e.g., $100 \mathrm{fs}$ ). Here, the UED measurements show more detailed changes in the observable as a function of pump-probe delay than the TRPES measurements. However, the direct information on the changing electronic energy and nonadiabatic coupling between electronic states available in the TRPES measurements provides an important perspective on the dynamics.

\section{CONCLUSION}

We have presented UED and TRPES measurements of the dynamics of $\mathrm{CH}_{2} \mathrm{I}_{2}$ following photoexcitation in the deep UV. The measurements combine structural and energetic probing of the dynamics and are compared with trajectory surface hopping calculations, yielding a detailed picture of the dynamics. We note that each experimental approach alone would miss some information. For example, while the TRPES measurements illuminate the internal conversion and dissociation of the molecule and suggest that the dissociation dynamics are nonlocal (multiple wave packets), they do not provide any information about the rotational dynamics and the complicated structural changes accompanying dissociation. This information is of broad significance, as chemical reactivity and molecular function depend not only on the distance between two different functional groups of the molecule but also on their relative orientation (as in, e.g., protein folding). On the other hand, the relativistic UED measurements do not provide direct information on the internal conversion (nonadiabatic coupling between electronic states) and energy conversion of the molecule as it dissociates. By combining the two approaches, we found direct experimental evidence for nonadiabatic coupled nuclear-electronic dynamics, nonlocal evolution of the nuclear wave function (different groups of trajectories that have very different structural and energetic evolutions as a function of time), and rotational motion of the molecule during dissociation, which highlights the threedimensional information available from diffractive imaging measurements. The combined measurements and calculations provide a more complete view of molecular dynamics during physical and chemical transformation than would be available from a single approach alone.

\section{ACKNOWLEDGMENTS}

We gratefully acknowledge support from the U.S. Department of Energy (DOE) under Awards No. DE-AC02-05-CH11231, No. DE-AC02-76SF00515, No. DE-SC0014170, and No. DEFG02-08ER15984 for carrying out the measurements, and from the National Science Foundation under Grant No. 1806294 for the interpretation of the measurements. This project was partly supported by the Government of Hungary and the European Regional Development Fund under Grant No. VEKOP-2.3.216-2017-00015. The computational results presented have been achieved in part using the Vienna Scientific Cluster (VSC). R. F. acknowledges support from the Engineering and Physical Sciences Research Council (EPSRC). Open access funding provided by University of Vienna.

\section{APPENDIX A: EXPERIMENTAL DETAILS}

\section{TRPES and UED experimental details}

In both the UED and TRPES experiments, the sample (Diiodomethane, 99\% purity) was purchased from SigmaAldrich and used without further purification. The spectra of the UV pump pulses (similar for both experiments, $\lambda_{0} \sim 265 \mathrm{~nm}, h \nu=4.65 \mathrm{eV}$ ) are shown together with the $\mathrm{CH}_{2} \mathrm{I}_{2}$ absorption spectrum in Fig. 6 [87].

The gas-phase UED and TRPES experimental apparatuses have been described in detail previously $[52,68]$. Here, we expand on the discussion in the main text to describe experimental conditions for the two measurements in some more detail. First, we describe the TRPES experiments, which were carried out with two separate apparatuses at two separate locations, with a variety of pump-pulse parameters in order to test the sensitivity of the measurements to the details of the pump pulse. In the one apparatus, gas-phase $\mathrm{CH}_{2} \mathrm{I}_{2}$ molecules, seeded in $\mathrm{He}$ through the use of a bubbler, were introduced into the electron VMI spectrometer setup by means of a pulsed molecular beam. The beam was produced by a $1-\mathrm{kHz}$ EvenLavie pulsed valve [88], which was heated to $60^{\circ} \mathrm{C}$ during the experiment in order to prevent clustering. Ion time-offlight (TOF) spectra indicated that there was no cluster

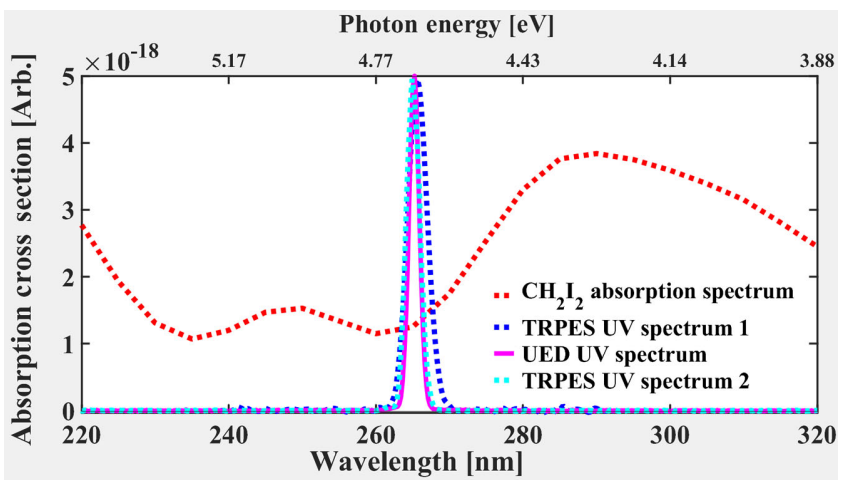

FIG. 6. $\mathrm{CH}_{2} \mathrm{I}_{2}$ absorption spectrum, together with the spectra for the pump pulses in the two experiments. The UV spectrum in the TRPES measurement is centered at $265.6 \mathrm{~nm}$, while the UV spectrum of the UED measurement is centered at $265.3 \mathrm{~nm}$. As the TRPES measurements were repeated with two different apparatuses, the spectrum for each of these pump pulses is shown separately. 
formation during the experiment. The molecular beam was expanded through a $250-\mu \mathrm{m}$ conical nozzle into a source chamber typically held at a base pressure of $10^{-6}$ Torr. The beam was then skimmed to yield a beam, with an estimated diameter of around $1 \mathrm{~mm}$, before entering an interaction chamber along the spectrometer TOF axis, typically held at a base pressure of $1 \times 10^{-8}$ Torr, and intersected, at $90^{\circ}$, by the copropagating pump and probe laser pulses. In the other TRPES apparatus, the molecules were introduced into the vacuum chamber as a room effusive beam. The measurements performed with the pulsed valve were carried out with the laser pulses timed to arrive at the peak of the Even-Lavie pulse and off the peak. These two measurements were compared with the effusive beam experiments. A detailed comparison of all three measurements indicated that there was no significant difference between the measured TRPES for molecules that were cooled in the supersonic expansion and ones that were at room temperature.

In both TRPES measurements, the probe pulses consisted of VUV light $(160 \mathrm{~nm}, 7.75 \mathrm{eV})$ generated through noncollinear four-wave mixing of the fundamental and third harmonic in an argon gas cell [71]. The details of this VUV generation setup can be found in Refs. [19,68]. Photoelectrons produced from the pump-probe laser interaction were velocity map imaged using electrostatic lenses onto a conventional microchannel plate (MCP) and phosphor-based (P47) detector setup. Images were recorded using a CCD camera. At each pump-probe delay step, 3000 laser shots were integrated to produce a VMI image, and the pump-probe scan was repeated 20 times. At the end of each individual scan, images were taken with UV and VUV pulses alone in order to subtract out background electrons. These background electrons can arise from either of the pulses scattering off of the chamber walls and the VMI plates or two-photon ionization of the molecules by VUV pulses.

In contrast with the TPRES measurements, the UED measurements made use of an ultrashort relativistic electron bunch as the probe. The electron bunch is generated by a radio-frequency (rf) electron gun, which is powered by an S-band Klystron, providing an $80-\mathrm{MV} / \mathrm{m}$ accelerating field. The electron energy can be tuned continuously up to $5 \mathrm{MeV}$. For our measurements, the kinetic energy was set to 3.7 $\mathrm{MeV}$, providing electrons with a speed of $0.993 \mathrm{c}$ (0.993 times the speed of light). Each electron bunch contains roughly $10^{4}$ electrons, and about 100 to 200 electrons are scattered from the molecular sample. The electron beam is focused to a diameter of $200 \mu \mathrm{m}$ FWHM, while the pump beam is focused down to a spot size of $300 \mu \mathrm{m}$ FWHM.

A $45^{\circ}$ mirror with a hole is used to combine the UV and electron beams. The electron bunch travels through the hole in the center, and the UV beam is reflected from the surface. The two beams propagate with a small angle $\left(<4^{\circ}\right)$ and intersect the gas jet roughly $250 \mu$ m under the nozzle of the pulsed valve. This small angle makes the velocity of the laser $0.998 \mathrm{c}$ along the electron beam direction, close to $0.993 \mathrm{c}$, the velocity of the electron beam. Therefore, the pump-probe delay smearing due to the velocity mismatch is on the order of 5 fs across the jet [53]. The size of the gas jet is roughly $300 \mu \mathrm{m}$ FWHM in the reaction region. The sample holder is heated up to $80^{\circ} \mathrm{C}$ in order to increase the vapor pressure. Helium is used as the carrier gas, and the backing pressure is set to 0.5 bar. The sample gas with $\mathrm{He}$ is then introduced into the vacuum chamber by a pulsed nozzle (Parker Hannifin Valve) with a $100-\mu \mathrm{m}$ opening. The nozzle is heated to $150{ }^{\circ} \mathrm{C}$ to avoid cluster generation.

The electron diffraction pattern is recorded with the detector $3.1 \mathrm{~m}$ downstream from the interaction region. The electron detector consists of a P43 phosphor screen with a center hole, a $45^{\circ}$ mirror with a hole in the center, an imaging lens, and an electron-multiplying charged-coupled device (EMCCD, Andor). The system runs at a repetition rate of $180 \mathrm{~Hz}$. The rough spatial and temporal overlap between the UV pump and electron beam probe is achieved by pump-probe measurements on a silicon film. In each pump-probe scan, a diffraction pattern at each time delay is integrated for 20 seconds, and the pump-probe scans are repeated 180 times.

\section{Data analysis details}

A detailed description of the photoelectron VMI image processing is provided in Ref. [73]. Most of the details of the UED data processing can be found in the Supplemental Material for Ref. [20].

Here, we provide further details on the analysis of the data to recover the $3 \mathrm{D}$ information. The $2 \mathrm{D}$ diffraction pattern measured in the UED experiments contains information about both the $3 \mathrm{D}$ molecular structure and the angular distribution of the molecular ensemble [89,90]. Figure 7 shows the raw diffraction pattern from the

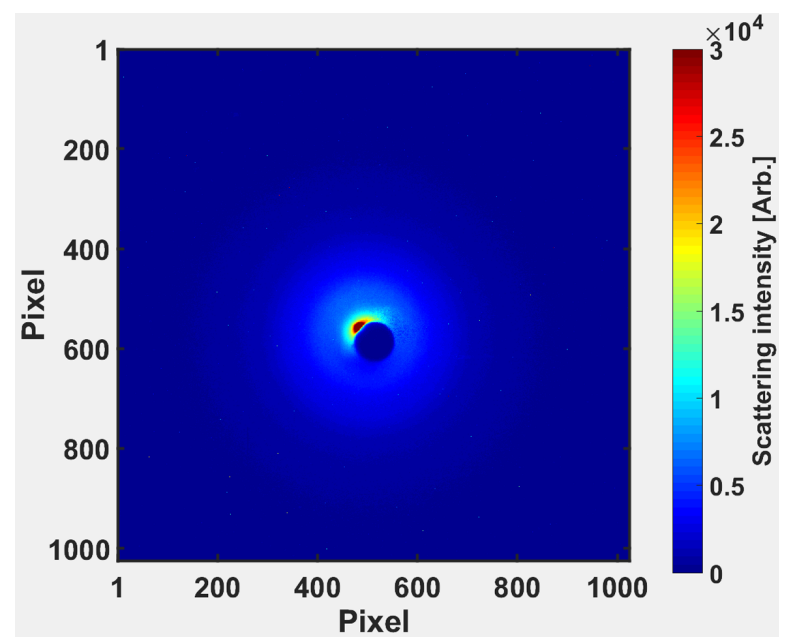

FIG. 7. Raw $\mathrm{CH}_{2} \mathrm{I}_{2}$ diffraction pattern averaged between 450 and $600 \mathrm{fs}$. The electron beam is directed to the top-left corner of the hole in the phosphor screen in order to access some of the signal in the low-s region. 

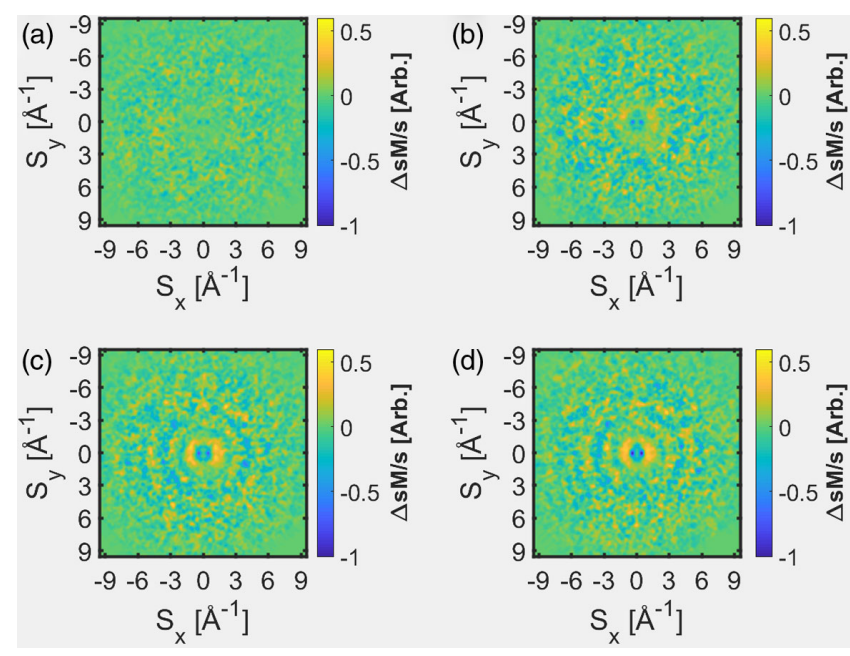

FIG. 8. Measured modified diffraction difference patterns$\Delta \mathrm{sM}(\mathrm{s})$. (a) Pattern averaged over delays from -250 to $-150 \mathrm{fs}$. (b) Pattern averaged over delays from -50 to 50 fs. (c) Pattern averaged over delays from 200 to 300 fs. (d) Pattern averaged over delays from 500 to $600 \mathrm{fs}$.

measurement. Because of the hole in the phosphor screen, the diffraction pattern is not available over a circular disk in the center of the camera with a radius of $2 \AA^{-1}$. We positioned the electron beam away from the center of the hole, so the diffraction center is located at the upper-left corner of the hole. This process allowed us to partially obtain the diffraction pattern down to $\mathrm{s}=0.5 \AA^{-1}$. Because of the cylindrical symmetry of the diffraction pattern around the pump-pulse polarization axis, we were able to partially fill in the difference diffraction pattern in the hole area with measurements from regions outside of the hole, making the hole region radius as small as $\mathrm{s}=0.5 \AA^{-1}$. Our simulations show that the diffraction difference decreases monotonically down to zero from $\mathrm{s}=0.5 \AA^{-1}$. We therefore took the value from the edge of the small hole region and assumed a linear decrease down to zero in the diffraction center. We tested the effect of this method using simulated diffraction patterns. It significantly decreased the high-frequency modulations in the large-pair distance region of the real space image as compared with doing nothing to the measurements in the hole region (i.e., leaving it to be a zero signal).

In order to access the three-dimensional features in the data, we first applied a 2D inverse Fourier transform to the diffraction difference pattern (difference between the diffraction pattern for positive and negative delays-shown for different delays in Fig. 8), which yields the projected pair distribution function difference in real space. By applying an inverse Abel transform, we obtain the 3D pair distribution function difference $(\triangle \mathrm{PDF})$. Because of the cylindrical symmetry, the $3 \mathrm{D}$ pair distribution difference is integrated over the azimuthal angle in order to obtain and plot the $\triangle \mathrm{PDF}$ as a function of pair distance $R$ and polar angle $\theta$ (relative to the laser polarization), shown in Fig. 9.

A few additional filters are applied to increase the signalto-noise ratio (SNR). These filters include a low pass filter in momentum space $\left(e^{-s^{2} / s_{\max }^{2}}\right.$, with $\left.s_{\max }=7 \AA^{-1}\right)$, zero padding before the Fourier transform, and Legendre polynomial fitting [48] (up to sixth order) followed by the inverse Abel transform.

Here, we assess the uncertainty of the time-zero determination in the two experimental measurements. In the TRPES experiment, we determine time zero of our system

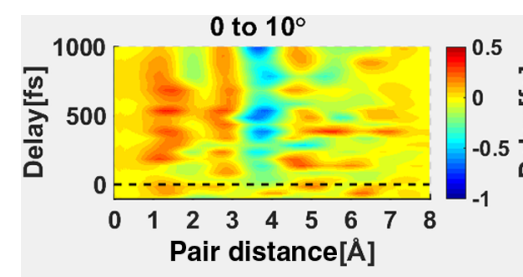

30 to $40^{\circ}$

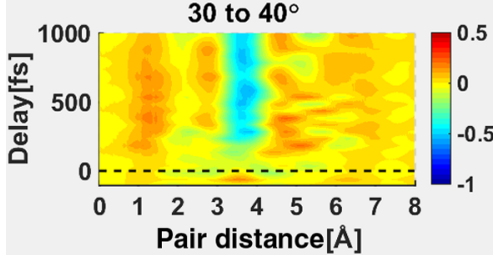

60 to $70^{\circ}$

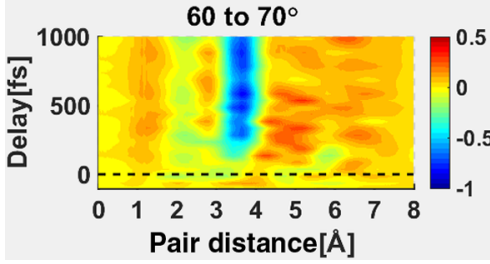

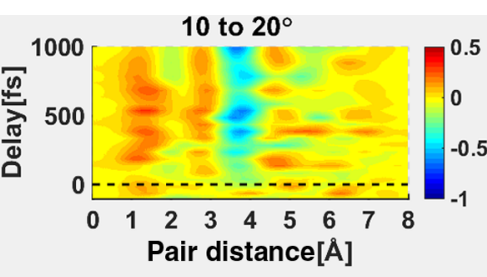

40 to $50^{\circ}$

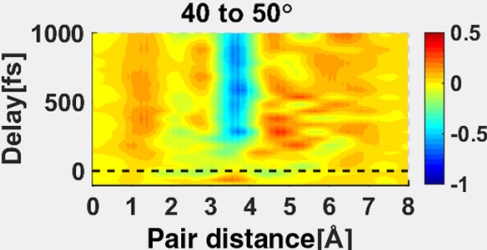

70 to $80^{\circ}$

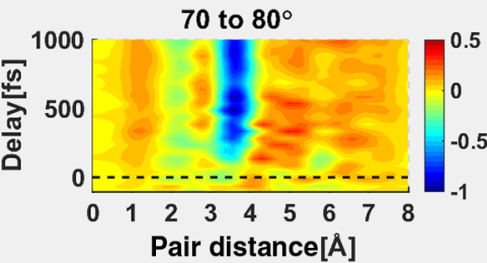

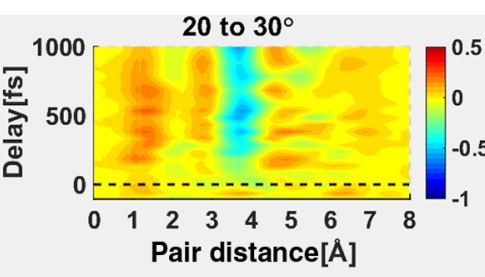

50 to $60^{\circ}$

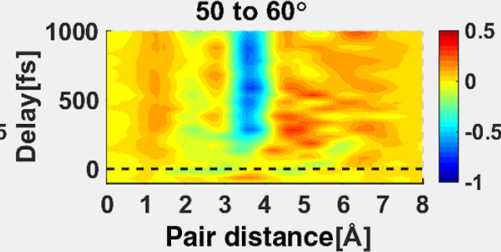

80 to $90^{\circ}$

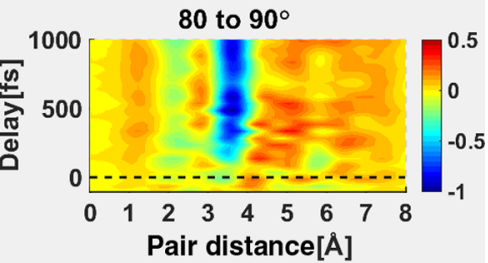

FIG. 9. Angle-dependent $\triangle \mathrm{PDF}$. Each panel shows the $\triangle \mathrm{PDF}$ within a $10^{\circ}$ polar angle region. The depletion in the I-I initial pair distance shows the largest amplitude at $90^{\circ}$, consistent with the fact that the transition dipole moment is strongest along the direction perpendicular to the I-I pair. Note that the angle dependence of $\triangle \mathrm{PDF}$ is different for different atomic pair distances. 


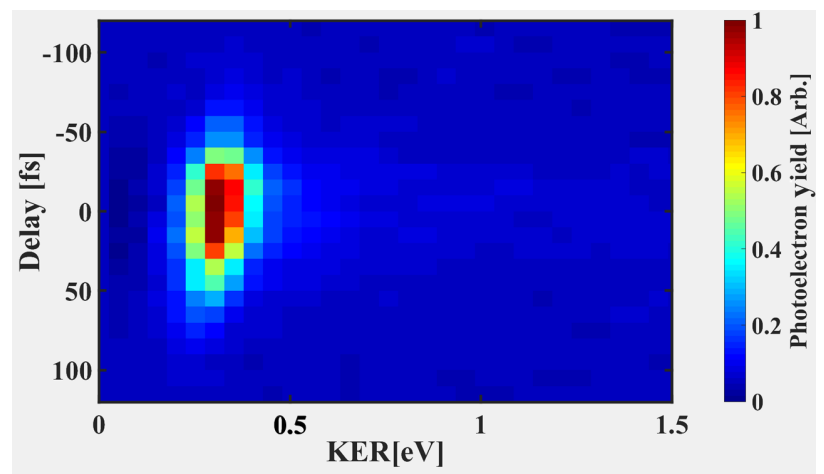

FIG. 10. TRPES for Xe used to determine time zero.

by looking at the nonresonant $1+1^{\prime}$ photon ionization of Xe. The TRPES is shown in Fig. 10. This method is also used to determine the impulse response function (IRF) of our system (measured to be about 75 fs full width at half maximum (FWHM). By fitting the Xe data to a Gaussian profile, we can determine time zero for the experiment. In the UED experiment, the IRF for the UED measurements is more difficult to measure, but it is estimated to be under 150 fs [20]. We performed a pump-probe measurement with a thin silicon film to locate time zero within a $300-\mathrm{fs}$ window. We improved the precision in the time-zero determination by comparing the phase of modulations in the measured $\triangle \mathrm{PDF}$ with those of modulations in the simulated $\triangle \mathrm{PDF}$ at $R=2.85 \AA$. Figure $4(\mathrm{~b})$ in the main text plots both the measured and simulated $\triangle \mathrm{PDF}$ at $2.85 \AA$. The simulation indicates that the first peak of the modulation occurs at around $135 \mathrm{fs}$. A higher-precision estimate of time zero is obtained by adjusting the experimental delay axis slightly such that data and simulation agree on the position of the peak. With this adjustment, we found that all of the subsequent oscillations in the $\triangle \mathrm{PDF}$ at $R=2.85 \AA$ agreed for simulation and experiment.

A bootstrapping method is used to calculate the statistical uncertainty for both the UED and TRPES measurements. In the UED experiment, 180 diffraction patterns are measured at each time delay. This data set is randomly resampled 100 times, with 180 images selected each time, using a standard bootstrapping method. This process generates 100 bootstrapped patterns. Each bootstrapped pattern is analyzed using the full data analysis routine, and the standard deviation from these 100 results is taken as the standard error. Figures 4(a)-4(c) show the bootstrapping analysis results at three different pair distances.

Figure 11 shows the bootstrapping analysis for the TRPES data. In the TRPES experiment, 20 photoelectron VMI images are measured at each time delay. This data set is randomly resampled 20 times, with 20 images selected each time, using a standard bootstrapping method. This process generates 20 bootstrapped VMI images. Each bootstrapped VMI image is analyzed with the same method in the TRPES data analysis, and the standard deviation is
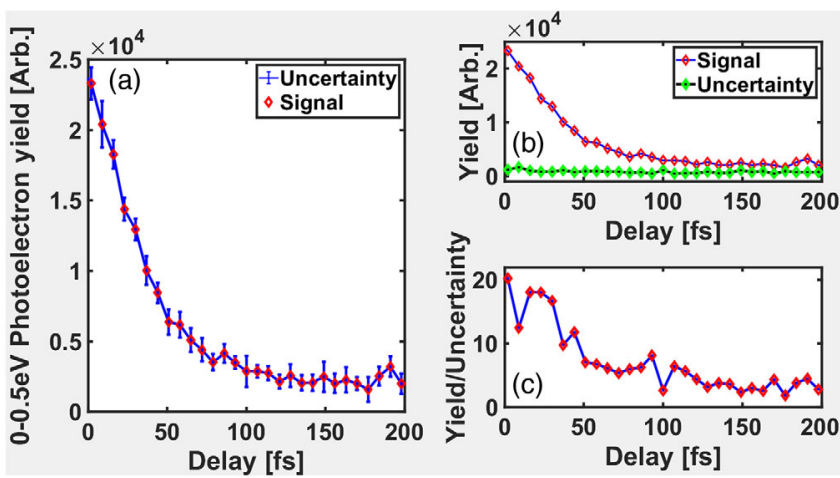

FIG. 11. Uncertainty analysis of low-energy photoelectron yield. Panel (a) shows the photoelectron yield as a function of pump-probe delay for electrons below $0.5 \mathrm{eV}$, along with the uncertainty (error bars), which is given by the standard deviation from the bootstrapping analysis. Panel (b) shows the yield and uncertainty as separate curves as a function of delay. Panel (c) shows the ratio of the yield and uncertainty, demonstrating a signal-to-noise ratio of greater than 5 over the relevant range of delays.

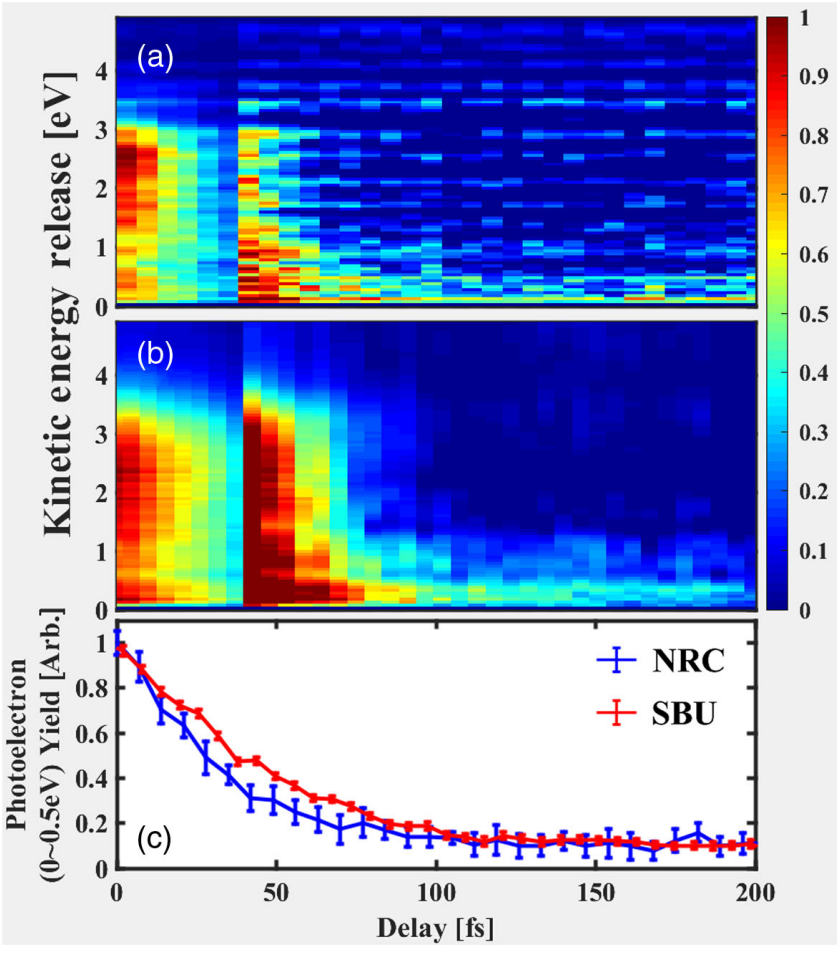

FIG. 12. Comparison of TRPES measurements carried out at two different locations with different pump-pulse parameters. (a) TRPES measurements carried out at the National Research Council in Canada (NRC) with a pump-pulse intensity of $0.5 \mathrm{TW} / \mathrm{cm}^{2}$ and a bandwidth of $2 \mathrm{~nm}$ FWHM. (b) TRPES measurements carried out at Stony Brook University (SBU) with a pump-pulse intensity of $1.0 \mathrm{TW} / \mathrm{cm}^{2}$ and a bandwidth of $1.5 \mathrm{~nm}$ FWHM. (c) Yield of low-energy electrons for the two measurements. In panels (a) and (b), both spectra are multiplied by a factor of 4 after $40 \mathrm{fs}$, as in Fig. 2. 
taken as the standard error. Specifically, we examine the region of the lower kinetic energy tail (between 0 and $0.5 \mathrm{eV}$ ), where we find that the signal is more than 5 times the standard deviation between 0 and 100 fs.

In order to assess the sensitivity of the TRPES measurements to the pump-pulse central frequency, bandwidth, and intensity, we performed a number of experiments where these parameters were varied. A representative measurement from experiments performed at Stony Brook University is shown together with a measurement performed at the National Research Council of Canada in Fig. 12. We found that the main features in the measurement were not sensitive to these variations within the range of parameters we considered (central wavelength between $266 \mathrm{~nm}$ and $262 \mathrm{~nm}$, bandwidth between $1.5 \mathrm{~nm}$ and $2.0 \mathrm{~nm}$, and intensity between $0.3 \mathrm{TW} / \mathrm{cm}^{2}$ and $1.5 \mathrm{TW} / \mathrm{cm}^{2}$ ).

\section{APPENDIX B: CALCULATION DETAILS}

\section{Trajectory surface hopping calculation}

Here, we describe the details in the calculations. In the trajectory surface hopping calculations, the IPEA (an empirical correction applied to the zeroth-order Hamiltonian) shift was set to zero, which was found to improve the results in combination with the small double- $\zeta$ basis set [91]. However, in order to avoid intruder states and ensure a stable propagation in the dynamics simulations, an imaginary shift of 0.3 Hartree was added [92]. In order to account for scalar-relativistic effects, the secondorder Douglas-Kroll-Hess (DKH) Hamiltonian [93] was employed, while spin-orbit couplings (SOCs) were computed with the RASSI [94] and AMFI [95] formalisms. The dynamics were run by employing the velocity-Verlet algorithm with a time step of $0.5 \mathrm{fs}$ for the nuclear dynamics and a time step of 0.02 fs for the propagation of the electronic wave function, using the "local diabatization" formalism [96]. Energy conservation during a surface hop was ensured by scaling of the full velocity vectors, which is an approximation compared to scaling along the nonadiabatic coupling vectors. This approximation was necessary here since the nonadiabatic coupling vectors were not available for our level of theory.

The transition probabilities between states of the same multiplicity are hence taken into account with the help of wave-function overlap calculations. We employed an energy-based decoherence correction with a parameter of 0.1 Hartree [97]. In our experience, more sophisticated approaches to decoherence correction, such as the augmented version of Subotnik and co-workers [98], do not improve the quality of the results in systems like the present ones, where the frequency of hopping is comparably low. The initial geometries and velocities for the trajectories were sampled from a Wigner distribution of the harmonic ground-state potential. In this way, 10000 geometries were produced for each molecule, and a single-point calculation at the MS-CASPT2 $(12,8)$ level of theory was performed at each of these geometries in order to obtain the state energies and oscillator strengths.

The initial excited-states were selected stochastically [99], restricting the excitation energy window to energies around our pump pulse. The ionization simulation was carried out as postprocessing for every fifth time step. Since initial wave functions were taken from the previous time step, intermittent points were recalculated with CASSCF. From each semiquantum trajectory, we can directly extract the time-resolved energy, state index, transition dipole moment (TDM) direction, and molecular geometry. We then made used of these semiquantum trajectory surface hopping calculation results to evaluate the measured observables in both TRPES and UED experimental methods. In these calculations, several trajectories crashed due to numerical instabilities in the CASPT2 calculations using numerical gradients. The measurement observables were all calculated based on the active trajectories for each time delay. Of the 158 trajectories that we made use of in our analysis, about half crashed before 250 fs. We performed detailed checks on the crashed trajectories to ensure that crashes did not occur at specific geometries or in specific electronic states, which could have introduced a systematic bias into the analysis of the remaining ones.

As a test of the accuracy of the calculated TRPES, particularly given the important role that spin-orbit coupling plays, we calculated the ground-state photoelectron spectrum both with and without spin-orbit coupling, and we compare the calculated results with the measured spectrum [100] in Fig. 13. The calculation results are shifted by about $0.3 \mathrm{eV}$ before plotting in order to compensate for the roughly $3 \%$ error in the ionization potential. As one can see,

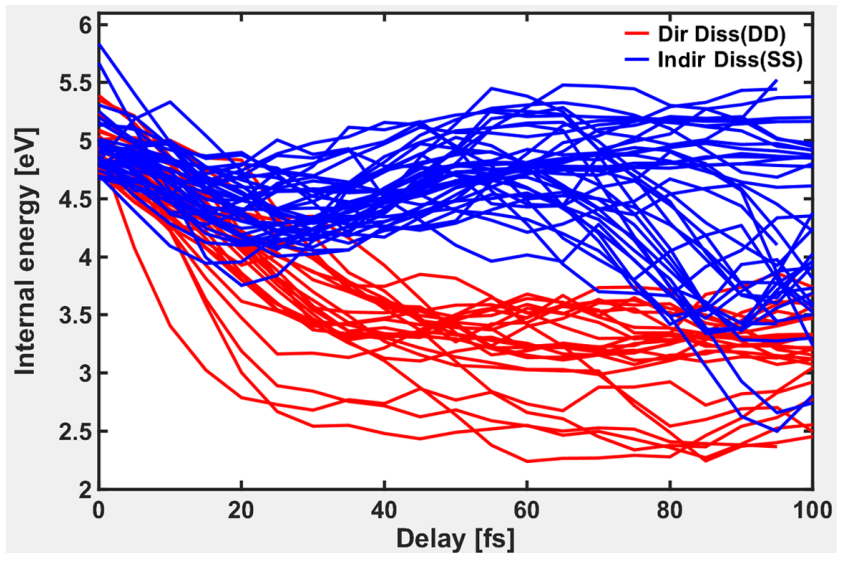

FIG. 13. Calculated energies as a function of time for individual trajectories in the first $100 \mathrm{fs}$. Each individual line represents a single trajectory. Direct and indirect trajectories are plotted in red and blue, respectively. DD represents direct dissociation, and SS represents the symmetric stretch associated with indirect trajectories. 
aside from a small shift in the overall energy, the calculation with spin-orbit coupling agrees with the measurements quite well (correct number of peaks, appropriate spacing, and relative heights), while the calculation without spin-orbit coupling does not produce the correct number of peaks or spacing between peaks. Our results are consistent with an extensive body of prior work, which makes use of the Dyson method for calculating photoelectron spectra [101-104]. The Dyson approach has also been used to calculate photoelectron spectra away from the FranckCondon point in several earlier time-resolved photoelectron spectroscopy studies $[18,19,81,105-107]$.

In order to assess whether the TRPES measurements are sensitive to nonadiabatic dynamics, we carried out calculations of the TRPES with and without surface hopping. Figure 14 compares the measurements with and without the surface hopping included in the calculations. In panels (a) and (b), it is clear that the features at long times between 0 and $2 \mathrm{eV}$ in the calculations without hopping are not present in the measurements. Panel (d) shows the energy-integrated photoelectron yield from the calculations together with the experimental measurements. The results of the calculations with surface hopping agree well with the experimental measurements, while the calculations without surface hopping show significant differences. This comparison highlights the nonadiabatic dynamics captured by the TRPES measurements.

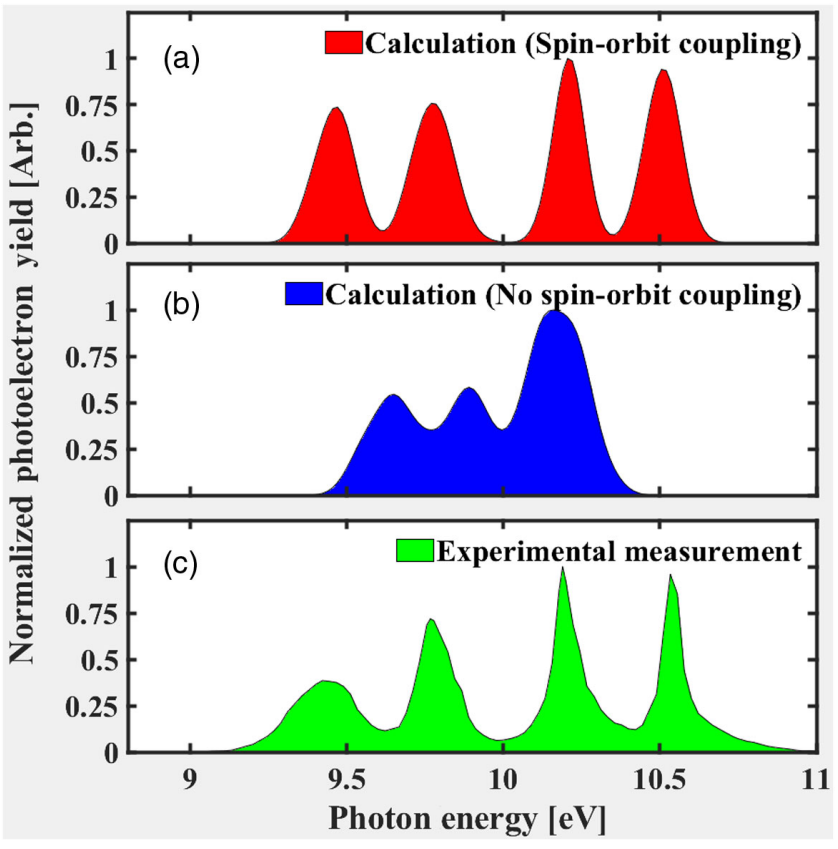

FIG. 14. Comparison between measured and calculated photoelectron spectra. (a) Calculated ground-state photoelectron spectrum including spin-orbit coupling. (b) Calculated ground-state photoelectron spectrum without spin-orbit coupling. (c) Replotted version of the measured photoelectron spectra from Ref. [100].

\section{Direct and indirect dissociative trajectories}

Figure 15 shows the potential energy of each individual trajectory as a function of time delay, with the direct dissociation trajectories shown in red and the indirect dissociation trajectories shown in blue. One can see that the trajectories naturally fall into two groups, with the direct dissociation trajectories losing energy more rapidly (in under $40 \mathrm{fs}$ ) and the indirect dissociation trajectories generally maintaining their energy until 80 fs or beyond.

This separation of trajectories into two groups can also be seen in the molecular geometry as a function of time. Figure 16 shows the absolute value of the difference between the $\mathrm{C}$-I bond lengths, $\Delta \mathrm{L}$, in the top two panels [(a) and (b)], as well as the I-C-I angle $\alpha$ in the bottom two panels [(c) and (d)]. Panels (a) and (c) show the individual trajectories, whereas panels (b) and (d) show the average for the two groups, with the standard deviation for each group indicated by the shading behind the lines. It is clear

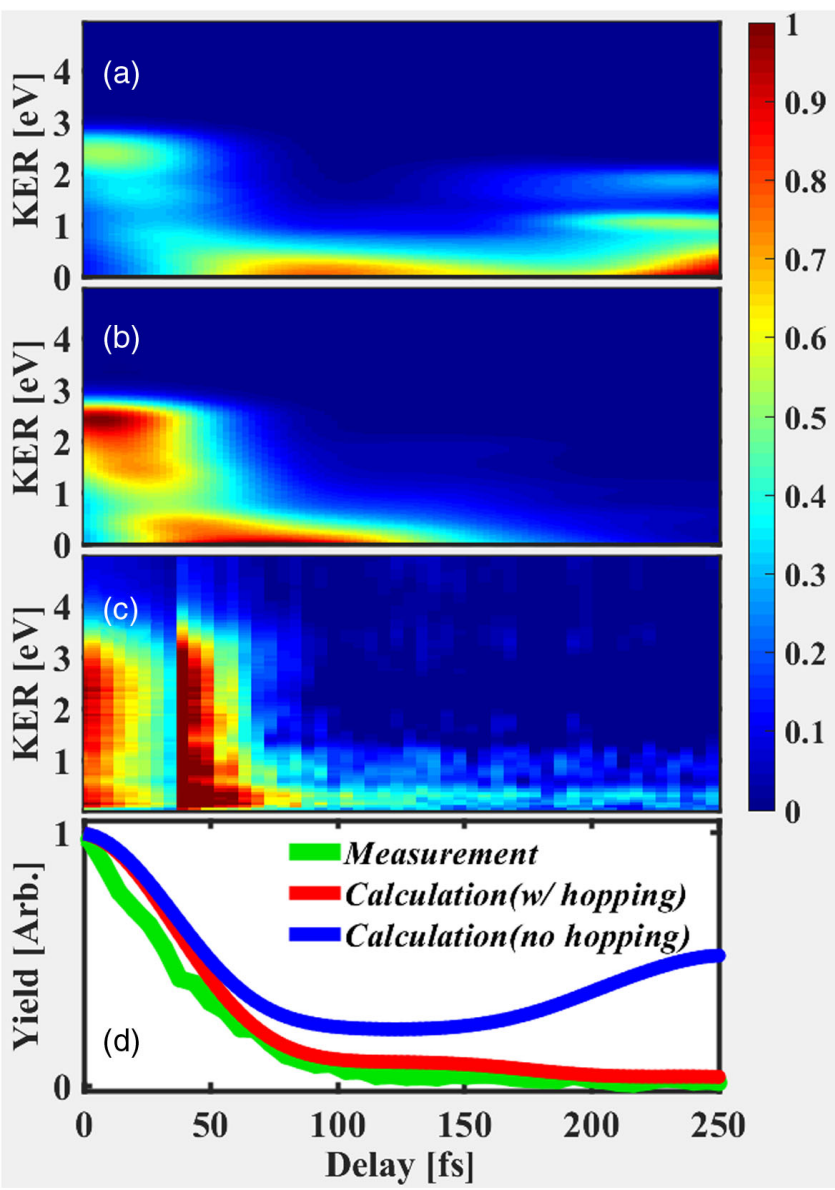

FIG. 15. Comparison between measured photoelectron spectra and calculations with or without surface hopping. (a) Calculated TRPES without surface hopping between different electronic states. (b) Calculated TRPES including surface hopping between different electronic states. (c) Measured TRPES. (d) Measured and calculated (with and without surface hopping) energyintegrated photoelectron yield as a function of pump-probe delay. 

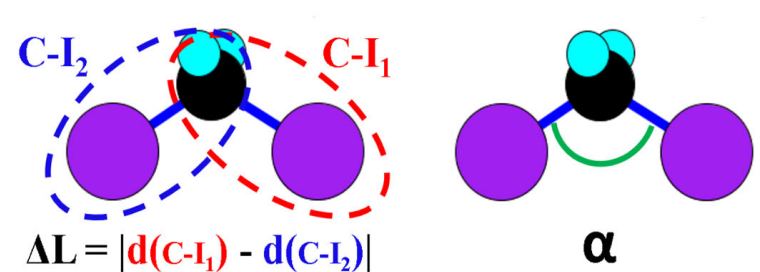

$\Delta \mathrm{L}=\left|\mathrm{d}\left(\mathrm{C}-\mathrm{I}_{1}\right)-\mathrm{d}\left(\mathrm{C}-\mathrm{I}_{2}\right)\right|$

$\boldsymbol{\alpha}$
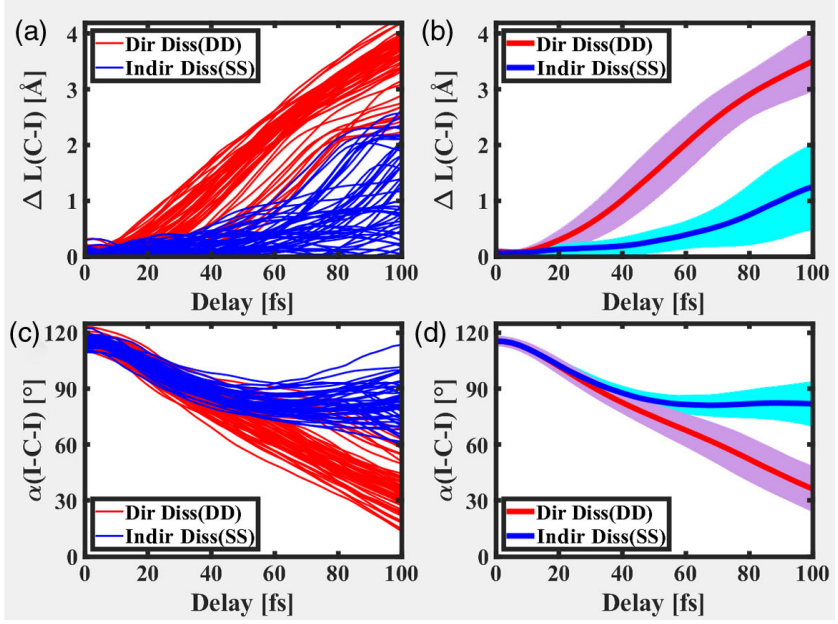

FIG. 16. Calculated C-I pair distances and I-C-I angles as a function of time in the first $100 \mathrm{fs}$. Two cartoons in the figure illustrate the quantities being plotted: the absolute difference between $\mathrm{C}$-I pair distances, $\Delta \mathrm{L}$, and the I-C-I angle $\alpha$. (a) Time evolution of $\Delta \mathrm{L}$ from 0 to $100 \mathrm{fs}$. Each line represents a single trajectory. Red and blue lines correspond to direct and indirect dissociation trajectories, respectively. (b) Time evolution of averaged $\Delta \mathrm{L}$ from trajectories in different groups. (c) Time evolution of $\alpha$ from 0 to 100 fs. (d) Time evolution of averaged $\alpha$ from trajectories in different groups. In panels (b) and (d), 1 standard deviation is treated as the error bar, which is indicated by the shaded region behind each line.

from the figure that the two groups are well defined, as they are separated by an amount that is larger than the standard deviation for each group.

In order to show that the rotation of the $\mathrm{CH}_{2}$ group leads to modulations in the $\triangle \mathrm{PDF}$ at half the rotational period, in Fig. 17 we show the I-C-I angle as a function of time up to $500 \mathrm{fs}$ for an ensemble of direct dissociation trajectories. As the pump pulse only aligns the molecules rather than orienting them, the projection of the C-I bond distance onto the plane of the detector repeats itself twice per rotational period rather than once for the excited molecular ensemble.

\section{Calculated pair distribution function difference}

In order to obtain the calculated TRPES, the ionization probability along each trajectory was evaluated by calculating the Dyson orbital norms. More details are available in Ref. [73]. For the case of UED, the time evolution of the molecular geometry along the trajectory was used to calculate the electron diffraction pattern at each delay.

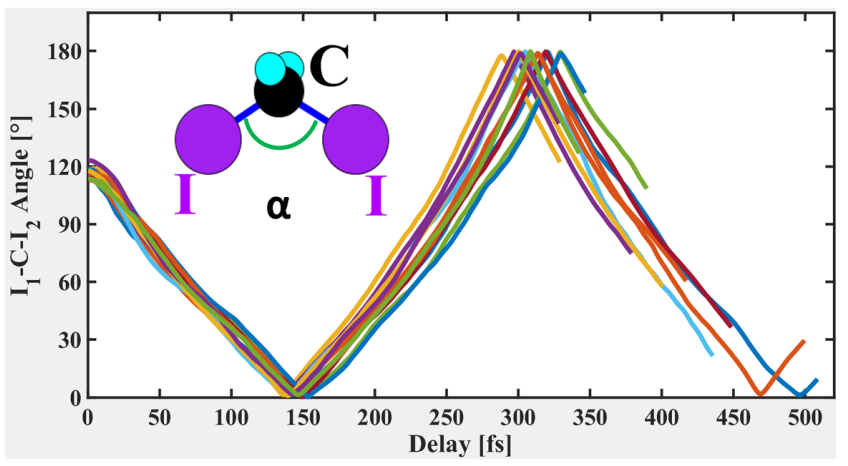

FIG. 17. I-C-I angle as a function of time for direct dissociation trajectories illustrating the rotation of the $\mathrm{CH}_{2}$ group as the $\mathrm{C}-\mathrm{I}$ bond is broken. A cartoon of the $\mathrm{CH}_{2} \mathrm{I}_{2}$ molecule is shown inside the figure.

In order to calculate the time-resolved pair distribution difference, we simulated the electron diffraction pattern according to molecular geometries extracted from the surface hopping calculations. The elastic electron scattering amplitudes for $\mathrm{C}, \mathrm{I}$, and $\mathrm{H}$ atoms were calculated using the Dirac partial-wave method (ELSEPA) [83]. We made use of the independent atom approximation in the scattering process, in which the scattering amplitude and phase of each individual atom in the molecule is approximated to be the same as an isolated atom. The electrons that participate in chemical bonds were neglected. Each trajectory has the positions for each atom as a function of time, as well as the TDM direction with respect to the relative positions of the atoms. Experimental conditions (electron beam energy, the hole in the phosphor screen, instrument response function, etc.) were applied to the simulated pattern in order to compare with the experimental measurements.

The scattering intensity for a single molecule with orientation $\vec{a}$ at time $t$ can be written as

$$
I^{\vec{a}}(t ; \vec{s})=\sum_{i=1}^{N} \sum_{j=1}^{N} f_{i}(s) f_{j}^{*}(s) \operatorname{Re}\left[e^{\left(i \vec{s} \cdot \vec{r}_{i j}(\vec{a} ; t)\right)}\right],
$$

where $N$ is the number of atoms in the molecule, $f_{i}(s)$ is the complex-valued elastic scattering amplitude for the $i$ th atom, and $\vec{r}_{i j}(\vec{a}, t)$ is the vector pointing from the $i$ th to the $j$ th atom. Here, $\vec{s}$ is the momentum transfer vector between the initial and final wave factor $\left(\vec{s}=\vec{k}-\overrightarrow{k_{0}}\right)$ with magnitude $(4 \pi / \lambda) \sin (\Theta / 2)$, where $\Theta$ denotes the electron scattering angle. The total scattering intensity for the molecular ensemble can be written as an incoherent sum of the singlemolecule scattering intensities,

$$
I(t ; \vec{s})=\sum_{\vec{a}} I^{\vec{a}}(t ; \vec{s}) .
$$

In our experiments, the linearly polarized UV light preferentially excites molecules whose TDM direction is 
along the laser polarization, leading to a $\cos ^{2}(\theta)$ dependence in which $\theta$ is the angle between laser polarization and TDM directions. In order to simulate the PDF using the trajectories from the surface hopping calculation, we took into account the proper angular distribution induced by the photoselection rules. In order to capture the angular distribution of the molecule during the excitation, we simulated an ensemble of diffraction patterns from an ensemble of different orientations of the molecule $[89,90]$. We defined a molecular frame $[X, Y, Z]$, in which we fixed the carbon atom at the origin and located the two iodine atoms in the $X-Y$ plane, with the $Y$ axis along the middle between the two iodine atoms. A lab frame $[x, y, z]$ is defined by the laser polarization direction along the $y$ axis and the electron beam traveling along the $z$ axis. Different orientations of the molecules in the lab frame were generated by starting with the lab and molecular frames being overlapped and rotating the molecular frame along the proper Euler angles, $\alpha, \beta, \gamma$, in which $\alpha$ represents a rotation around the $z$ axis, $\beta$ represents a rotation around the rotated $x$ axis, and $\gamma$ represents the rotation around the rotated $z$ axis.

The angle $\theta$ between the TDM and the laser polarization was taken into account by weighing the scattering magnitude with $\cos ^{2}(\theta)$. The diffraction pattern of the total scattering intensity is then a sum from all the rotated geometries, which can be written as

$$
I(t ; \vec{s})=\sum_{\alpha} \sum_{\beta} \sum_{\gamma} I^{(\alpha, \beta, \gamma)}(t ; \vec{s}) \cos ^{2} \theta_{(\alpha, \beta, \gamma)} .
$$

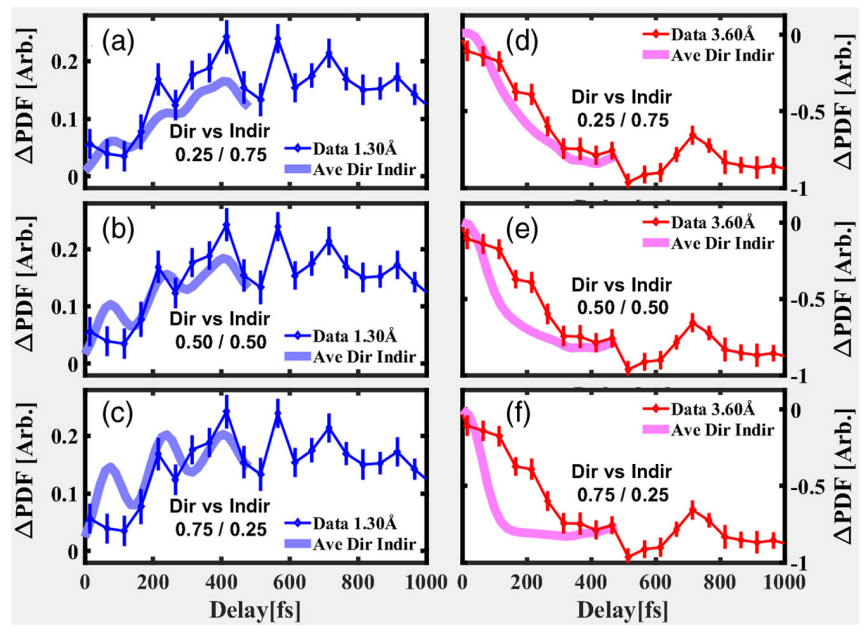

FIG. 18. Analysis of direct and indirect trajectory weighting. Panels (a) and (d) show the measured and calculated $\triangle P D F$ at $1.30 \AA$ and $3.60 \AA$, respectively, for a $0.25 / 0.75$ weighting of direct vs indirect trajectories in the average. Panels (b) and (e) show the measured and calculated $\triangle \mathrm{PDF}$ at $1.30 \AA$ and $3.60 \AA$, respectively, for a $0.50 / 0.50$ weighting of direct vs indirect trajectories in the average. Panels (c) and (f) show the measured and calculated $\Delta \mathrm{PDF}$ at $1.30 \AA$ and $3.60 \AA$, respectively, for a $0.75 / 0.25$ weighting of direct vs indirect trajectories in the average.
The diffraction pattern was calculated for the geometry at each time delay of the trajectories, and once the diffraction pattern for the ensemble was simulated, the same diffraction pattern analysis routine that was used for the experimental data was applied to the simulated diffraction patterns to generate the $\triangle \mathrm{PDF}$.

\section{Analysis of direct and indirect trajectory averaging}

Here, we provide a brief analysis of the weighting of direct and indirect trajectories when comparing their average to the measurements. Figure 18 compares the measurements and calculations for different weightings of direct and indirect trajectories. The figure shows that the measurements and simulations agree best for a direct/ indirect mixture between $0.25 / 0.75$ and $0.50 / 0.50$.

\section{APPENDIX C: SEPARATION OF ONE- AND TWO-PHOTON-ABSORPTION DRIVEN DYNAMICS}

Here, we provide details on how we separated one- and two-photon contributions to the UED and TRPES measurements. In both experiments, we worked at the lowest pump-pulse fluence where we could measure the dynamics with a sufficient signal-to-noise ratio to extract meaningful information from the measurements. The energy of the measured photoelectrons in the TRPES measurements were consistent with the absorption of only one pump and one probe photon, assuming ionization to low-lying states of the molecular cation. While one could, in principle, produce similar energy photoelectrons by absorbing two pump photons and ionizing to higher lying states of the molecular cation, this case is less likely and was ruled out by performing several measurements with different pumppulse fluence.

The situation is more complicated for the UED measurements. UED has relatively low sensitivity compared to most spectroscopic techniques. Lower pumping fluence leads to low SNR, a low scattering range in momentum transfer space, an inability to transform to real space, etc. These aspects can be significantly improved with higher pump fluence. However, higher pump fluence can lead to multiphoton absorption. In our case, it is more difficult to detect excited-state dynamics without pumping hard enough to induce some two-photon absorption.

In order to check that our measurements were not dominated by multiphoton absorption from the pump pulse, we performed measurements of the diffraction signal as a function of the pump-pulse intensity. Figure 19 shows the strongest feature in the momentum space diffraction pattern, i.e., the difference signal between 1.1 and $2.8 \AA^{-1}$, as a function of pump-pulse intensity. The measurements described in the main text were performed for a pump-pulse intensity of about $1 \mathrm{TW} / \mathrm{cm}^{2}$, which corresponds to the linear portion of the graph shown in Fig. 19. In order to 


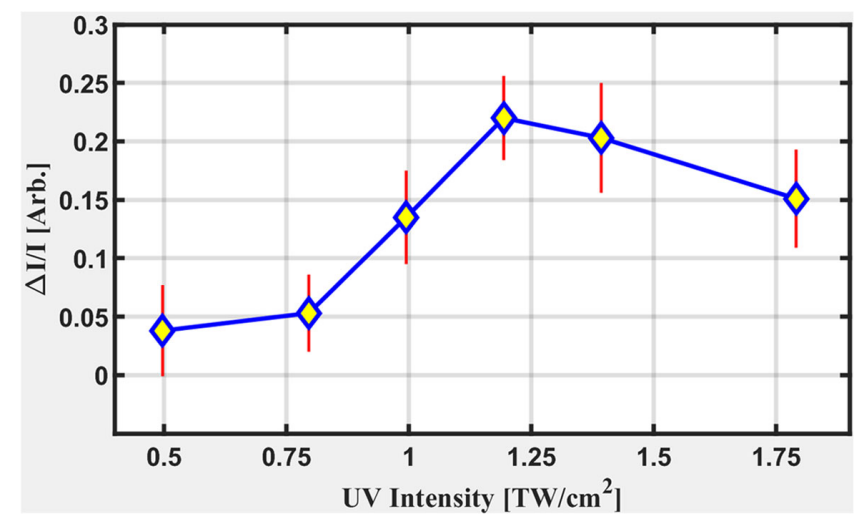

FIG. 19. UV pump-pulse intensity dependence. The ratio of the diffraction pattern difference, $\Delta \mathrm{I} / \mathrm{I}$, between 1.1 and $2.8 \AA^{-1}$ is plotted as a function of UV pump-pulse intensity. The delay was chosen between 250 and $300 \mathrm{fs}$. All data shown in the main text were taken at an intensity of around $1 \mathrm{TW} / \mathrm{cm}^{2}$.

further evaluate the relative contributions of multiphoton vs one-photon absorption to the measured dynamics, we performed two additional analyses.

In the first approach, we compared the modulations in the signal at $2.85 \AA$ with the depletion at $3.60 \AA$ in order to evaluate the two-photon driven dynamics. The simulations of the two-photon driven dynamics indicate that the wave packet is compact and oscillates, leading to a minimum I-I distance of $2.85 \AA$, and the simulations of the one-photon driven dynamics do not make any contribution to the $\triangle \mathrm{PDF}$ at $2.85 \AA$. Given that both dynamics lead to a decrease in the PDF at a pair distance of $3.60 \AA$ while only the twophoton driven dynamics contribute to the modulations at $2.85 \AA$, we made use of the ratio of the $\triangle \mathrm{PDF}$ at these two distances to estimate the fraction of molecules absorbing two pump photons vs those absorbing one. According to Figs. 4(b) and 4(c), the oscillation amplitude at $2.85 \AA$ is about $12 \%$ of the total depletion of $\triangle \mathrm{PDF}$ at $3.60 \AA$, a few hundred fs after the pump pulse. This result indicates that the two-photon absorption contribution to the dynamics is about an order of magnitude lower than the one-photon absorption contribution, consistent with the linear response to the pump pulse shown in Fig. 19.

A second approach makes use of the angular dependence of the measured diffraction signal $[23,89]$. We fit the angledependent three-dimensional $\triangle \mathrm{PDF}$ to a series of Legendre polynomials in order to obtain the coefficients for each even order (the so-called $\beta$ parameters). One-photon absorption should only contribute to second- and zeroth-order Legendre polynomials $\left(\beta_{2}\right.$ and $\beta_{0}$ ), while two-photon absorption contributes to fourth-, second-, and zeroth-order Legendre polynomials $\left(\beta_{4}, \beta_{2}\right.$, and $\left.\beta_{0}\right)$. In general, an $M$-photon process will contribute to all the even orders from $\beta_{0}$ up to $\beta_{2 M}$; thus, determining $\beta_{M}$ places a limit on the contribution of $M$-photon absorption. Such a $\beta$ parameter analysis is attractive because one can have a model-independent check of the data to quantitatively determine how much one-, two-, and three-photon processes contribute to the signal. Applying this analysis to our simulations confirmed that two-photon absorption driven Rydberg dynamics contributed to $\beta_{0}, \beta_{2}$, and $\beta_{4}$, while one-photon absorption driven valence dynamics contributed to only $\beta_{0}$ and $\beta_{2}$.

As with the simulations, we applied the $\beta$ parameter analysis to the measured $\Delta \mathrm{PDF}$ up to sixth order. While the time and $R$-dependent $\beta_{0}$ and $\beta_{2}$ parameters match the overall features with those from the simulated results of one-photon dynamics, $\beta_{4}$ and $\beta_{6}$ are large and fluctuate. This result could be due to higher order $(M>1)$ photon absorption driven dynamics, or it could be due to the noise in the data [30], as simulated data that we analyzed led to large $\beta_{4}$ and $\beta_{6}$ parameters in the fitting if we introduced noise into the simulated data set. In order to determine whether the large $\beta_{4}$ and $\beta_{6}$ parameters were due to noise or multiphoton absorption, we compared the $\beta_{4}$ and $\beta_{6}$ parameters for positive and negative delays. For negative delays, the $\beta_{4}$ and $\beta_{6}$ values should be noise driven, whereas for positive delays, they could contain contributions from multiphoton absorption driven dynamics. Thus, the ratio of their values for positive and negative delays provides some measure of whether multiphoton absorption plays a role in the measured dynamics. A ratio greater than 1 indicates that there are pump-pulse induced dynamics that lead to a given $\beta$ parameter order, whereas a low ratio $(\leq 1)$ means that the $\beta$ parameter is largely noise driven.

We focused our analysis on the $\mathrm{R}$ region of the $\triangle \mathrm{PDF}$ associated with I-I depletion since all orders of multiphoton absorption should contribute if there are any dynamics that involve motion of the I atoms. Figure 20 shows the positive/ negative delay ratio for the $\beta$ parameters as a function of order. Both $\beta_{0}$ and $\beta_{2}$ show values of the ratio much larger than 1 , while the ratios for $\beta_{4}$ and $\beta_{6}$ show very low values. This analysis confirms that our measurements are dominated by one-photon absorption driven dynamics.

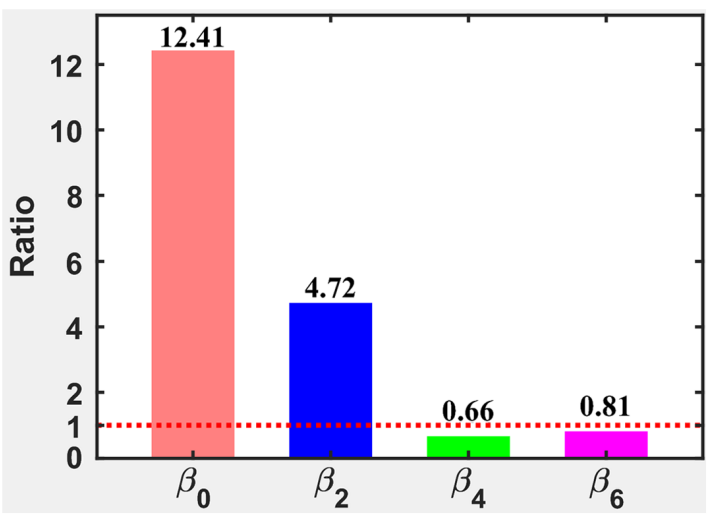

FIG. 20. $\quad \beta$ parameter analysis of measured $\triangle$ PDF. Ratio of integrated $\beta$ parameter values for positive and negative time delays are plotted in the figure with $\beta$ order of $0,2,4$, and 6 . Each $\beta$ parameter is obtained by integration between $3.2 \AA$ and $4.0 \AA$. A red dotted line indicates a ratio of 1 . 
[1] C. E. Crespo-Hernndez, B. Cohen, P. M. Hare, and B. Kohler, Ultrafast Excited-State Dynamics in Nucleic Acids, Chem. Rev. 104, 1977 (2004).

[2] D. Polli, P. Altoè, O. Weingart, K. M. Spillane, C. Manzoni, D. Brida, G. Tomasello, G. Orlandi, P. Kukura, R. A. Mathies, M. Garavelli, and G. Cerullo, Conical Intersection Dynamics of the Primary Photoisomerization Event in Vision, Nature (London) 467, 440 (2010).

[3] Y.-C. Cheng and G. R. Fleming, Dynamics of Light Harvesting in Photosynthesis, Annu. Rev. Phys. Chem. 60, 241 (2009).

[4] H.-J. Son, S. Jin, S. Patwardhan, S. J. Wezenberg, N. C. Jeong, M. So, C. E. Wilmer, A. A. Sarjeant, G. C. Schatz, R. Q. Snurr, O. K. Farha, G. P. Wiederrecht, and J. T. Hupp, Light-Harvesting and Ultrafast Energy Migration in Porphyrin-Based Metal-Organic Frameworks, J. Am. Chem. Soc. 135, 862 (2013).

[5] X. Li, L. E. Sinks, B. Rybtchinski, and M. R. Wasielewski, Ultrafast Aggregate-to-Aggregate Energy Transfer within Self-Assembled Light-Harvesting Columns of Zinc Phthalocyanine Tetrakis(perylenediimide), J. Am. Chem. Soc. 126, 10810 (2004).

[6] R. Berera, R. van Grondelle, and J. T. M. Kennis, Ultrafast Transient Absorption Spectroscopy: Principles and Application to Photosynthetic Systems, Photosynth. Res. 101, 105 (2009).

[7] T. Weinacht and B. J. Pearson, Time-Resolved Spectroscopy: An Experimental Perpective (CRC Press, 2018).

[8] J. Sheng and J.Z. Zhang, Dissociative Chemisorption of $\mathrm{H}_{2}$ on Ni Surface: Time-Dependent Quantum Dynamics Calculation and Comparison with Experiment, J. Chem. Phys. 96, 3866 (1992).

[9] C. Meier and V. Engel, Electron Kinetic Energy Distributions from Multiphoton Ionization of $\mathrm{Na}_{2}$ with Femtosecond Laser Pulses, Chem. Phys. Lett. 212, 691 (1993).

[10] A. Hofmann and R. de Vivie-Riedle, Adiabatic Approach for Ultrafast Quantum Dynamics Mediated by Simultaneously Active Conical Intersections, Chem. Phys. Lett. 346, 299 (2001).

[11] H. Tamura, S. Nanbu, T. Ishida, and H. Nakamura, Ab Initio Nonadiabatic Quantum Dynamics of Cyclohexadiene/Hexatriene Ultrafast Photoisomerization, J. Chem. Phys. 124, 084313 (2006).

[12] R. Improta, V. Barone, A. Lami, and F. Santoro, Quantum Dynamics of the Ultrafast $\pi \pi * / n \pi *$ Population Transfer in Uracil and 5-Fluoro-Uracil in Water and Acetonitrile, J. Phys. Chem. B 113, 14491 (2009).

[13] O. Carstensen, J. Sielk, J. B. Schönborn, G. Granucci, and B. Hartke, Unusual Photochemical Dynamics of a Bridged Azobenzene Derivative, J. Chem. Phys. 133, 124305 (2010).

[14] A. Humeniuk, M. Wohlgemuth, T. Suzuki, and R. Mitrić, Time-Resolved Photoelectron Imaging Spectra from NonAdiabatic Molecular Dynamics Simulations, J. Chem. Phys. 139, 134104 (2013).

[15] S. L. Horton, Y. Liu, P. Chakraborty, P. Marquetand, T. Rozgonyi, S. Matsika, and T. Weinacht, Strong-FieldVersus Weak-Field-Ionization Pump-Probe Spectroscopy, Phys. Rev. A 98, 053416 (2018).
[16] H. R. Hudock, B. G. Levine, A. L. Thompson, H. Satzger, D. Townsend, N. Gador, S. Ullrich, A. Stolow, and T. J. Martinez, $A b$ Initio Molecular Dynamics and TimeResolved Photoelectron Spectroscopy of Electronically Excited Uracil and Thymine, J. Phys. Chem. A 111, 8500 (2007).

[17] S. P. Neville, Y. Wang, A. E. Boguslavskiy, A. Stolow, and M.S. Schuurman, Substituent Effects on Dynamics at Conical Intersections: Allene and Methyl Allenes, J. Chem. Phys. 144, 014305 (2016).

[18] W. J. Glover, T. Mori, M. S. Schuurman, A. E. Boguslavskiy, O. Schalk, A. Stolow, and T. J. Martínez, Excited State NonAdiabatic Dynamics of the Smallest Polyene, Trans 1, 3-Butadiene. II. Ab Initio Multiple Spawning Simulations, J. Chem. Phys. 148, 164303 (2018).

[19] M. R. Coates, M. A. Larsen, R. Forbes, S. P. Neville, A. E. Boguslavskiy, I. Wilkinson, T. I. Sølling, R. Lausten, A. Stolow, and M. S. Schuurman, Vacuum Ultraviolet Excited State Dynamics of the Smallest Ring, Cyclopropane. II. Time-Resolved Photoelectron Spectroscopy and Ab Initio Dynamics, J. Chem. Phys. 149, 144311 (2018).

[20] J. Yang et al., Imaging $\mathrm{CF}_{3} \mathrm{I}$ Conical Intersection and Photodissociation Dynamics with Ultrafast Electron Diffraction, Science 361, 64 (2018).

[21] T. J. A. Wolf et al., The Photochemical Ring-Opening of 1,3-Cyclohexadiene Imaged by Ultrafast Electron Diffraction, Nat. Chem. 11, 504 (2019).

[22] J. Cao, H. Ihee, and A. H. Zewail, Ultrafast Electron Diffraction and Direct Observation of Transient Structures in a Chemical Reaction, Proc. Natl. Acad. Sci. U.S.A. 96, 338 (1999).

[23] A. Stolow, A. E. Bragg, and D. M. Neumark, Femtosecond Time-Resolved Photoelectron Spectroscopy, Chem. Rev. 104, 1719 (2004).

[24] R. Berera, R. van Grondelle, and J. T. Kennis, Ultrafast Transient Absorption Spectroscopy: Principles and Application to Photosynthetic Systems, Photosynth. Res. 101, 105 (2009).

[25] C. Ruckebusch, M. Sliwa, P. d. Pernot, A. De Juan, and R. Tauler, Comprehensive Data Analysis of Femtosecond Transient Absorption Spectra: A Review, J. Photochem. Photobiol. C 13, 1 (2012).

[26] C. Wan, T. Fiebig, S. O. Kelley, C. R. Treadway, J. K. Barton, and A.H. Zewail, Femtosecond Dynamics of DNA-Mediated Electron Transfer, Proc. Natl. Acad. Sci. U.S.A. 96, 6014 (1999).

[27] J.-M. L. Pecourt, J. Peon, and B. Kohler, Ultrafast Internal Conversion of Electronically Excited RNA and DNA Nucleosides in Water, J. Am. Chem. Soc. 122, 9348 (2000).

[28] W. J. Schreier, T. E. Schrader, F. O. Koller, P. Gilch, C. E. Crespo-Hernández, V. N. Swaminathan, T. Carell, W. Zinth, and B. Kohler, Thymine Dimerization in DNA Is an Ultrafast Photoreaction, Science 315, 625 (2007).

[29] V. Blanchet, M. Z. Zgierski, T. Seideman, and A. Stolow, Discerning Vibronic Molecular Dynamics Using TimeResolved Photoelectron Spectroscopy, Nature (London) 401, 52 (1999).

[30] K. L. Reid, Photoelectron Angular Distributions, Annu. Rev. Phys. Chem. 54, 397 (2003). 
[31] S. Ullrich, T. Schultz, M.Z. Zgierski, and A. Stolow, Electronic Relaxation Dynamics in DNA and RNA Bases Studied by Time-Resolved Photoelectron Spectroscopy, Phys. Chem. Chem. Phys. 6, 2796 (2004).

[32] A. Stolow and J. G. Underwood, Time-Resolved Photoelectron Spectroscopy of Nonadiabatic Dynamics in Polyatomic Molecules, Adv. Chem. Phys. 139, 497 (2008).

[33] T. Suzuki, Time-Resolved Photoelectron Spectroscopy of Non-Adiabatic Electronic Dynamics in Gas and Liquid Phases, Intl. Rev. Phys. Chem. 31, 265 (2012).

[34] P. Sándor, V. Tagliamonti, A. Zhao, T. Rozgonyi, M. Ruckenbauer, P. Marquetand, and T. Weinacht, Strong Field Molecular Ionization in the Impulsive Limit: Freezing Vibrations with Short Pulses, Phys. Rev. Lett. 116, 063002 (2016).

[35] V. Tagliamonti, P. Sándor, A. Zhao, T. Rozgonyi, P. Marquetand, and T. Weinacht, Nonadiabatic Dynamics and Multiphoton Resonances in Strong-Field Molecular Ionization with Few-Cycle Laser Pulses, Phys. Rev. A 93, 051401(R) (2016).

[36] R. Forbes, A. E. Boguslavskiy, I. Wilkinson, J. G. Underwood, and A. Stolow, Excited State Wavepacket Dynamics in $\mathrm{NO}_{2}$ Probed by Strong-Field Ionization, J. Chem. Phys. 147, 054305 (2017).

[37] J.C. Williamson and A. H. Zewail, Ultrafast Electron Diffraction. 4. Molecular Structures and Coherent Dynamics, J. Phys. Chem. 98, 2766 (1994).

[38] M. Dantus, S. B. Kim, J. C. Williamson, and A. H. Zewail, Ultrafast Electron Diffraction. 5. Experimental Time Resolution and Applications, J. Phys. Chem. 98, 2782 (1994).

[39] J. C. Williamson, J. Cao, H. Ihee, H. Frey, and A. H. Zewail, Clocking Transient Chemical Changes by Ultrafast Electron Diffraction, Nature (London) 386, 159 (1997).

[40] P. Reckenthaeler, M. Centurion, W. Fuß, S. A. Trushin, F. Krausz, and E. E. Fill, Time-Resolved Electron Diffraction from Selectively Aligned Molecules, Phys. Rev. Lett. 102, 213001 (2009).

[41] C. J. Hensley, J. Yang, and M. Centurion, Imaging of Isolated Molecules with Ultrafast Electron Pulses, Phys. Rev. Lett. 109, 133202 (2012).

[42] M. Centurion, P. Reckenthaeler, F. Krausz, and E. Fill, Picosecond Electron Diffraction from Molecules Aligned by Dissociation, J. Mol. Struct. 978, 141 (2010).

[43] P. Zhang, J. Yang, and M. Centurion, Tilted Femtosecond Pulses for Velocity Matching in Gas-Phase Ultrafast Electron Diffraction, New J. Phys. 16, 083008 (2014).

[44] J. Cao and K. R. Wilson, Ultrafast X-Ray Diffraction Theory, J. Phys. Chem. A 102, 9523 (1998).

[45] K. Gaffney and H. Chapman, Imaging Atomic Structure and Dynamics with Ultrafast X-Ray Scattering, Science 316, 1444 (2007).

[46] F. Schotte, M. Lim, T. A. Jackson, A. V. Smirnov, J. Soman, J. S. Olson, G. N. Phillips, M. Wulff, and P. A. Anfinrud, Watching a Protein as It Functions with 150-ps Time-Resolved X-Ray Crystallography, Science 300, 1944 (2003).

[47] M. P. Minitti, J. M. Budarz, A. Kirrander, J. S. Robinson, D. Ratner, T. J. Lane, D. Zhu, J. M. Glownia, M. Kozina,
H. T. Lemke, M. Sikorski, Y. Feng, S. Nelson, K. Saita, B. Stankus, T. Northey, J. B. Hastings, and P. M. Weber, Imaging Molecular Motion: Femtosecond X-Ray Scattering of an Electrocyclic Chemical Reaction, Phys. Rev. Lett. 114, 255501 (2015).

[48] J. M. Glownia et al., Time-Resolved Pump-Probe Experiments at the LCLS, Opt. Express 18, 17620 (2010).

[49] J. Küpper et al., X-Ray Diffraction from Isolated and Strongly Aligned Gas-Phase Molecules with a FreeElectron Laser, Phys. Rev. Lett. 112, 083002 (2014).

[50] M. R. Ware, J. M. Glownia, A. Natan, J. P. Cryan, and P. H. Bucksbaum, On the Limits of Observing Motion in TimeResolved X-Ray Scattering, Phil. Trans. R. Soc. A 377, 20170477 (2019).

[51] M. Ware, J. M. Glownia, A. Natan, J. Cryan, and P. Bucksbaum, Fourier-Transform Inelastic X-Ray Scattering: A New Kind of Gas-Phase Vibrational Spectroscopy, in Conference on Lasers and Electro-Optics, OSA Technical Digest (Optical Society of America, 2018), paper no. FM4F.5.

[52] J. Yang, M. Guehr, X. Shen, R. Li, T. Vecchione, R. Coffee, J. Corbett, A. Fry, N. Hartmann, C. Hast, K. Hegazy, K. Jobe, I. Makasyuk, J. Robinson, M. S. Robinson, S. Vetter, S. Weathersby, C. Yoneda, X. Wang, and M. Centurion, Diffractive Imaging of Coherent Nuclear Motion in Isolated Molecules, Phys. Rev. Lett. 117, 153002 (2016).

[53] J. Yang et al., Diffractive Imaging of a Rotational Wavepacket in Nitrogen Molecules with Femtosecond Megaelectronvolt Electron Pulses, Nat. Commun. 7, 11232 (2016).

[54] J. P. F. Nunes and M. Centurion, Ultrafast and ThreeDimensional Diffractive Imaging of Isolated Molecules with Electron Pulses, Adv. At. Mol. Opt. Phys. 68, 39 (2019).

[55] S.P. Weathersby et al., Mega-Electron-Volt Ultrafast Electron Diffraction at SLAC National Accelerator Laboratory, Rev. Sci. Instrum. 86, 073702 (2015).

[56] J. C. Tully, Molecular Dynamics with Electronic Transitions, J. Chem. Phys. 93, 1061 (1990).

[57] F. Allum, M. Burt, K. Amini, R. Boll, H. Köckert, P. K. Olshin, S. Bari, C. Bomme, F. Brauße, B. C. de Miranda et al., Coulomb Explosion Imaging of $\mathrm{Ch}_{3} \mathrm{I}$ and $\mathrm{Ch}_{2} \mathrm{ClI}$ Photodissociation Dynamics, J. Chem. Phys. 149, 204313 (2018).

[58] M. Burt et al., Coulomb-Explosion Imaging of Concurrent $\mathrm{CH}_{2}$ BrI Photodissociation Dynamics, Phys. Rev. A 96, 043415 (2017).

[59] B. W. Toulson, J. P. Alaniz, J. G. Hill, and C. Murray, Near-UV Photodissociation Dynamics of $\mathrm{CH}_{2} \mathrm{I}_{2}$, Phys. Chem. Chem. Phys. 18, 11091 (2016).

[60] Y.-J. Liu, L. De Vico, R. Lindh, and W.-H. Fang, SpinOrbit Ab Initio Investigation of the Ultraviolet Photolysis of Diiodomethane, Chem. Phys. Chem. 8, 890 (2007).

[61] M. Odelius, M. Kadi, J. Davidsson, and A. N. Tarnovsky, Photodissociation of Diiodomethane in Acetonitrile Solution and Fragment Recombination into Iso-Diiodomethane Studied with Ab Initio Molecular Dynamics Simulations, J. Chem. Phys. 121, 2208 (2004).

[62] X. Zheng and D. L. Phillips, Solvation Effects on the Iodoform Ultraviolet Direct Photodissociation Reaction: 
Opening the Photoisomerization Channel, Chem. Phys. Lett. 324, 175 (2000).

[63] J. Zhang, E. J. Heller, D. Huber, D. G. Imre, and D. Tannor, $\mathrm{CH}_{2} \mathrm{I}_{2}$ Photodissociation: Dynamical Modeling, J. Chem. Phys. 89, 3602 (1988).

[64] S. Roszak, W. Koski, J. Kaufman, and K. Balasubramanian, Structures and Electron Attachment Properties of Halomethanes $\left(\mathrm{CX}_{n} \mathrm{Y}_{m}, X=H, F ; Y=\mathrm{Cl}, B r, I ; n=0,4\right.$; $m=4 n)$, SAR and QSAR in environmental research 11, 383 (2001).

[65] M. Kawasaki, S. Lee, and R. Bersohn, Photodissociation of Molecular Beams of Methylene Iodide and Iodoform, J. Chem. Phys. 63, 809 (1975).

[66] A. Mandal, P. J. Singh, A. Shastri, and B. Jagatap, Electronic State Spectroscopy of Diiodomethane $\left(\mathrm{CH}_{2} \mathrm{I}_{2}\right)$ : Experimental and Computational Studies in the 30000-95000 $\mathrm{cm}^{-1}$ Region, J. Chem. Phys. 140, 194312 (2014).

[67] H. Xu, Y. Guo, S. Liu, X. Ma, D. Dai, and G. Sha, Photodissociation Dynamics of $\mathrm{CH}_{2} \mathrm{I}_{2}$ Molecules in the Ultraviolet Range Studied by Ion Imaging, J. Chem. Phys. 117, 5722 (2002).

[68] R. Forbes, V. Makhija, K. Veyrinas, A. Stolow, J. W. L. Lee, M. Burt, M. Brouard, C. Vallance, I. Wilkinson, R. Lausten, and P. Hockett, Time-Resolved Multi-Mass Ion Imaging: Femtosecond UV-VUV Pump-Probe Spectroscopy with the PimMS Camera, J. Chem. Phys. 147, 013911 (2017).

[69] A. T. Eppink and D. H. Parker, Velocity Map Imaging of Ions and Electrons Using Electrostatic Lenses: Application in Photoelectron and Photofragment Ion Imaging of Molecular Oxygen, Rev. Sci. Instrum. 68, 3477 (1997).

[70] M. Beutler, M. Ghotbi, F. Noack, and I. Hertel, Generation of Sub-50-fs Vacuum Ultraviolet Pulses by Four-Wave Mixing in Argon, Opt. Lett. 35, 1491 (2010).

[71] M. Ghotbi, M. Beutler, and F. Noack, Generation of $2.5 \mu \mathrm{J}$ Vacuum Ultraviolet Pulses with Sub-50 fs Duration by Noncollinear Four-Wave Mixing in Argon, Opt. Lett. 35, 3492 (2010).

[72] T. Kobayashi, T. Horio, and T. Suzuki, Ultrafast Deactivation of the $\pi \pi *(V)$ State of Ethylene Studied Using Sub20 fs Time-Resolved Photoelectron Imaging, J. Phys. Chem. A 119, 9518 (2015).

[73] S. L. Horton, Y. Liu, R. Forbes, V. Makhija, R. Lausten, A. Stolow, P. Hockett, P. Marquetand, T. Rozgonyi, and T. Weinacht, Excited State Dynamics of $\mathrm{CH}_{2} \mathrm{I}_{2}$ and $\mathrm{CH}_{2}$ BrI Studied with UV Pump VUV Probe Photoelectron Spectroscopy, J. Chem. Phys. 150, 174201 (2019).

[74] J. Yang, M. Guehr, T. Vecchione, M. S. Robinson, R. Li, N. Hartmann, X. Shen, R. Coffee, J. Corbett, A. Fry et al., Femtosecond Gas Phase Electron Diffraction with $\mathrm{MeV}$ Electrons, Faraday Discuss. 194, 563 (2016).

[75] X. Shen, R. Li, U. Lundstrm, T. Lane, A. Reid, S. Weathersby, and X. Wang, Femtosecond Mega-ElectronVolt Electron Microdiffraction, Ultramicroscopy 184, 172 (2018).

[76] X. Shen, R. Li, X. Wang, S. Weathersby, J. Yang et al., Ultrafast Mega-Electron-Volt Gas-Phase Electron Diffraction at SLAC National Accelerator Laboratory, in 9th International Particle Accelerator Conference
(IPAC'18), Vancouver, BC, Canada, 2018 (JACOW Publishing, Geneva, Switzerland, 2018), pp. 556-559.

[77] R. Li, M. Hoffmann, E. Nanni, S. Glenzer, M. Kozina, A. Lindenberg, B. Ofori-Okai, A. Reid, X. Shen, S. Weathersby et al., Terahertz-based subfemtosecond Metrology of Relativistic Electron Beams, Phys. Rev. Accel. Beams 22, 012803 (2019).

[78] S. Mai, M. Richter, M. Heindl, M. F. S. J. Menger, A. Atkins, M. Ruckenbauer, F. Plasser, M. Oppel, P. Marquetand, and L. González, SHARC2.0: Surface Hopping Including Arbitrary Couplings Program Package for NonAdiabatic Dynamics, sharc-md.org (2018).

[79] M. Richter, P. Marquetand, J. González-Vázquez, I. Sola, and L. González, SHARC: Ab Initio Molecular Dynamics with Surface Hopping in the Adiabatic Representation Including Arbitrary Couplings, J. Chem. Theory Comput. 7, 1253 (2011).

[80] F. Aquilante, J. Autschbach, R. K. Carlson, L. F. Chibotaru, M. G. Delcey, L. De Vico, N. Ferré, L. M. Frutos, L. Gagliardi, M. Garavelli et al., MOLCAS8: New Capabilities for Multiconfigurational Quantum Chemical Calculations Across the Periodic Table, J. Comput. Chem. 37, 506 (2016).

[81] M. Ruckenbauer, S. Mai, P. Marquetand, and L. González, Revealing Deactivation Pathways Hidden in TimeResolved Photoelectron Spectra, Sci. Rep. 6, 35522 (2016).

[82] F. Plasser, M. Ruckenbauer, S. Mai, M. Oppel, P. Marquetand, and L. González, Efficient and Flexible Computation of Many-Electron Wave Function Overlaps, J. Chem. Theory Comput. 12, 1207 (2016).

[83] F. Salvat, A. Jablonski, and C. J. Powell, Elsepadirac Partial-Wave Calculation of Elastic Scattering of Electrons and Positrons by Atoms, Positive Ions and Molecules, Comput. Phys. Commun. 165, 157 (2005).

[84] H. Ihee, J. Cao, and A. Zewail, Ultrafast Electron Diffraction: Structures in Dissociation Dynamics of $\mathrm{Fe}(\mathrm{CO})_{5}$, Chem. Phys. Lett. 281, 10 (1997).

[85] D. Geiler, B. J. Pearson, and T. Weinacht, Wave Packet Driven Dissociation and Concerted Elimination in $\mathrm{CH}_{2} \mathrm{I}_{2}$, J. Chem. Phys. 127, 204305 (2007).

[86] S. R. Nichols, T. C. Weinacht, T. Rozgonyi, and B. J. Pearson, Strong-Field Phase-Dependent Molecular Dissociation, Phys. Rev. A 79, 043407 (2009).

[87] H. Keller-Rudek, G. Moortgat, R. Sander, and R. Sörensen, The MPI-Mainz UV/VIS Spectral Atlas of Gaseous Molecules of Atmospheric Interest, Earth System Sci. Data 5, 365 (2013).

[88] U. Even, The Even-Lavie Valve as a Source for High Intensity Supersonic Beam, EPJ Techn. Instrum. 2, 17 (2015).

[89] J. S. Baskin and A. H. Zewail, Ultrafast Electron Diffraction: Oriented Molecular Structures in Space and Time, Chem. Phys. Chem. 6, 2261 (2005).

[90] J. S. Baskin and A. H. Zewail, Oriented Ensembles in Ultrafast Electron Diffraction, Chem. Phys. Chem. 7, 1562 (2006).

[91] J. P. Zobel, J. J. Nogueira, and L. González, The IPEA dilemma in CASPT2, Chem. Sci. 8, 1482 (2017). 
[92] N. Forsberg and P.-A. Malmqvist, Multiconfiguration Perturbation Theory with Imaginary Level Shift, Chem. Phys. Lett. 274, 196 (1997).

[93] M. Reiher, Relativistic Douglas-Kroll-Hess Theory, WIREs Comput. Mol. Sci. 2, 139 (2012).

[94] P. A. Malmqvist, B. O. Roos, and B. Schimmelpfennig, The Restricted Active Space (RAS) State Interaction Approach with Spin-Orbit Coupling, Chem. Phys. Lett. 357, 230 (2002).

[95] B. Schimmelpfennig, AMFI-An Atomic Mean Field Integral Program, Stockholm University (1996).

[96] G. Granucci, M. Persico, and A. Toniolo, Direct Semiclassical Simulation of Photochemical Processes with Semiempirical Wave Functions, J. Chem. Phys. 114, 10608 (2001).

[97] G. Granucci and M. Persico, Critical Appraisal of the Fewest Switches Algorithm for Surface Hopping, J. Chem. Phys. 126, 134114 (2007).

[98] A. Jain, E. Alguire, and J.E. Subotnik, An Efficient, Augmented Surface Hopping Algorithm that Includes Decoherence for Use in Large-Scale Simulations, J. Chem. Theory Comput. 12, 5256 (2016).

[99] M. Barbatti, G. Granucci, M. Persico, M. Ruckenbauer, M. Vazdar, M. Eckert-Maksić, and H. Lischka, The Onthe-Fly Surface-Hopping Program System Newton-X: Application to Ab Initio Simulation of the Nonadiabatic Photodynamics of Benchmark Systems, J. Photochem. Photobiol. A 190, 228 (2007).

[100] A. Potts, H. Lempka, D. Streets, and W. Price, Photoelectron Spectra of the Halides of Elements in Groups III, IV, V and VI, Phil. Trans. R. Soc. A 268, 59 (1970).

[101] C. M. Oana and A. I. Krylov, Dyson Orbitals for Ionization from the Ground and Electronically Excited States within Equation-of-Motion Coupled-Cluster Formalism: Theory,
Implementation, and Examples, J. Chem. Phys. 127, 234106 (2007).

[102] M. Ruckenbauer, S. Mai, P. Marquetand, and L. González, Photoelectron Spectra of 2-Thiouracil, 4-Thiouracil, and 2, 4-Dithiouracil, J. Chem. Phys. 144, 074303 (2016).

[103] E. Hossain, S. M. Deng, S. Gozem, A. I. Krylov, X.-B. Wang, and P. G. Wenthold, Photoelectron Spectroscopy Study of Quinonimides, J. Am. Chem. Soc. 139, 11138 (2017).

[104] M. A. Parkes, J. Crellin, A. Henley, and H. H. Fielding, A Photoelectron Imaging and Quantum Chemistry Study of the Deprotonated Indole Anion, Phys. Chem. Chem. Phys. 20, 15543 (2018).

[105] H. Tao, T. Allison, T. Wright, A. Stooke, C. Khurmi, J. Van Tilborg, Y. Liu, R. Falcone, A. Belkacem, and T. Martinez, Ultrafast Internal Conversion in Ethylene. I. The Excited State Lifetime, J. Chem. Phys. 134, 244306 (2011).

[106] J. A. Sánchez-Rodríguez, A. Mohamadzade, S. Mai, B. Ashwood, M. Pollum, P. Marquetand, L. González, C. E. Crespo-Hernández, and S. Ullrich, 2-Thiouracil Intersystem Crossing Photodynamics Studied by WavelengthDependent PhotoElectron and Transient Absorption Spectroscopies, Phys. Chem. Chem. Phys. 19, 19756 (2017).

[107] S. Mai, A. Mohamadzade, P. Marquetand, L. González, and S. Ullrich, Simulated and Experimental Time-Resolved Photoelectron Spectra of the Intersystem Crossing Dynamics in 2-Thiouracil, Molecules 23, 2836 (2018).

[108] See Supplemental Material at http://link.aps.org/ supplemental/10.1103/PhysRevX.10.021016 for $\mathrm{CH}_{2} \mathrm{I}_{2}$ time-dependent molecular geometry and pair distribution function for direct and indirect dissociation trajectories. 